\title{
From the hive to the table: Nutrition value, digestibility and bioavailability of the dietary phytochemicals present in the bee pollen and bee bread
}

\author{
Volkan Aylanc, Soraia I. Falcão, Seymanur Ertosun, Miguel Vilas-Boas \\ Centro de Investigação de Montanha (CIMO), Instituto Politécnico de Bragança, Campus de Santa Apolónia, 5300-253, Bragança, Portugal
}

\section{A R T I C L E I N F O}

\section{Keywords:}

Bee pollen

Bee bread

Dietary phytochemicals

Digestibility and bioavailability

Safety status

\begin{abstract}
A B S T R A C T
Background: Consumption of natural products has increased significantly due to the idea that if nutrition improves, this leads to improved health, general well-being and reduces the risk of developing certain diseases. Bee products, especially bee pollen (BP) and bee bread (BB), have demonstrated several nutritional and bioactive properties, which make them functional foods par excellence. Thus, understanding the digestibility and the changes of phytochemicals along the digestive tract, which give BP and BB the functional food attribute, is crucial.

Scope and approach: This review describes the digestibility, bioavailability, and absorption behaviors of dietary phytochemicals present in BP and BB. It also addresses possible factors that may adversely affect the human health due to its intake and highlights food practices for the industry.

Key findings and conclusions: Many studies have been conducted on BP and BB, which mostly evaluated the nutritional values and the bioactive compounds. However, few studies have addressed the nutritional and phytochemical content of BP and BB after digestion. Topics such as changes in the digestive tract, post-digestive bioaccessibility, tissue absorption scores and the degree of presence in the circulatory system of the phytochemicals that provide strong biological properties to $\mathrm{BP}$ and $\mathrm{BB}$, should be taken into consideration in future researches.
\end{abstract}

\section{Introduction}

The collection of bee products by humans is evidenced in rock paintings from ancient times and historical finds, showing that apiculture has a very old history. Mesolithic rock painting from around 7000 BC at Spider Caves (Cuevas de la Araña) located in Valencia (Spain) and wall painting on the Ancient Egyptian temple dating back to $2400 \mathrm{BC}$ highlight the importance of beehive products (Mizrahi \& Lensky, 2013). Ancient people used honey and pollen to treat many diseases, including wounds, ulcer and bowel problems (Mizrahi \& Lensky, 2013). Despite this traditional use of bee products, apiculture began appropriately when people learned to construct models in beekeeping and preserve the future of bee colonies with particular care and control. In a broader definition, apiculture became the maintenance of honey bees in hives and its management, oriented for honey production and for pollination, as well as the collection of other bee products (bee pollen, bee bread, beeswax, propolis, royal jelly and bee venom) and live material such as queen bees, swarms and packaged bees (Formato \& Smulders, 2011). Although important developments were made in beekeeping practice and bee products since the 16th century (Crane, 1992), the true origins of bee products were not known until a few centuries ago and their detailed chemical composition was only determined in the late $1900 \mathrm{~s}$ (Mizrahi \& Lensky, 2013).

The development and advancement of civilizations lead to new lifestyles and innovative food trends. The diet is shaped by various factors such as regional traditions, socioeconomic factors, cultural and educational activities and wellbeing. Indeed, there is an intense relationship between diseases and the composition of consumed foods. Proper and adequate diet has a significant effect on physical and mental development, at the same time that it has a protective effect against many diseases. Recently, special interest in bee pollen (BP) and bee bread (BB) has been given with the detailed definition and revelation of the nutritional values and therapeutic properties of these bee products (Gardana, Del Bo, Quicazán, Corrrea, \& Simonetti, 2018; Khalifa et al., 2020; Kieliszek et al., 2018; Mărgăoan et al., 2019; Thakur \& Nanda, 2020). Both BP and BB are characterized by their high nutritional value, containing macro and micronutrients, phenolic acids and polyphenols (Kieliszek et al., 2018).

Since both bee products contain almost all of the metabolic necessary

\footnotetext{
* Corresponding author.

E-mail address: mvboas@ipb.pt (M. Vilas-Boas).
} 


\begin{tabular}{|ll|}
\hline \multicolumn{2}{|l|}{ Abbreviations } \\
BB & bee bread \\
BP & bee pollen \\
CBG & cytosolic $\beta$-glucosidase \\
GIT & gastrointestinal tract \\
LPH & lactase phlorizin hydrolase \\
ROS & reactive oxygen species \\
TFC & total flavonoid content \\
TPC & total phenolic content \\
\hline
\end{tabular}

nutrients and phytochemicals, their directly or indirectly use in foods has an important therapeutic and pharmacological role in the prevention or reduction of several diseases (Bogdanov, 2011). Many studies have been conducted on BP and BB obtained in different geographic areas throughout the world. In general, they focused on microbiology, physicochemical properties, plant origin, extraction methods, biological and its therapeutic activities, but it lacks a detailed discussion on the nutritional value, digestibility and bioavailability of the phytochemicals in BP and BB. Therefore, the aim of this work is a comprehensive review of the nutritional value, digestibility and bioavailability of dietary phytochemicals in $\mathrm{BP}$ and $\mathrm{BB}$, as well as their absorption. Besides that, this review assesses the fundamental knowledge of $\mathrm{BP}$ and $\mathrm{BB}$ consumption and safety, and its potential for the food industry.

\section{Bee pollen and bee bread}

Bee pollen is known as one of the oldest nutritional supplements in history and contains almost all nourishing components for a diet (Bakour, Fernandes, Barros, Sokovic, \& Ferreira, 2019). Pollens are units of male gametophytes of flowering plants. Bees mix the pollen grains with their own secretions after collecting it from the flowers; this process allows pollen to moisten and become pellets; then these pellets stick to the pollen basket on the hind leg of the bees and are transported to the hive (Campos et al., 2008). This new product, which bees collect from flowers and combine with their own secretions, is called bee pollen. The $\mathrm{BP}$ is the background source of nutrients necessary for honey bee to grow during their larvae stage and to develop sufficiently during their youth (Tomás, Falcão, Russo-Almeida, \& Vilas-Boas, 2017) at the same time it presents important nutritional and therapeutic characteristics for humans (Denisow \& Denisow-Pietrzyk, 2016). Beekeepers place pollen traps at the entrance of hives to retain the BP transported by bees, enabling the commercial collection of pollen, Fig. 1.

$\mathrm{BP}$ is often characterized by its high protein content, rich nutritional value and a good source of bioactive compounds, all varying accordingly to its botanical origin, geographical and climatic characteristics (Mayda, Özkök, Bayram, Gerçek, \& Sorkun, 2020). It contains protein and amino acids $(10-40 \% \mathrm{w} / \mathrm{w})$, lipids $(1-13 \% \mathrm{w} / \mathrm{w})$, vitamins $(0.02-0.7 \%)$, several minerals ( $\mathrm{K}, \mathrm{P}, \mathrm{Mg}, \mathrm{Ca}, \mathrm{Na}, \mathrm{S}, \mathrm{Fe}, \mathrm{Cu}$, etc.), and significant phenolic acids and polyphenols (Kieliszek et al., 2018). Nevertheless, carbohydrates are the main components of BP composing $13-55 \%$ of dry weight (Campos, Frigerio, Lopes, \& Bogdanov, 2010), which includes polysaccharides, oligosaccharides and dietary fiber. For example, cellulose, an important polysaccharide, has a content in pollen of about $3-4 \%$ being the main component of the layers of pollen grains, and its presence significantly affects the digestibility of BP (Kieliszek et al., 2018).

BP can present different colors, such as white-black, brown, yellow, orange, yellow-blue, or yellow-brown, depending on the plants visited by bees (Campos et al., 2008). Pollen has a variable general appearance, normally with a spherical shape and diameters ranging from $0.01 \mathrm{~mm}$ to $0.1 \mathrm{~mm}$ (Ibrahim, Balasundram, Abdullah, Alias, \& Mardan, 2012). A bee colony can collect between $50 \mathrm{~g}$ and $250 \mathrm{~g}$ of pollen per day, and $15-40 \mathrm{~kg}$ of pollen per year (Thakur \& Nanda, 2020), with the worldwide production of pollen being approximately 1500 tons per year (Kieliszek et al., 2018).

Once inside the beehive, the BP is stored in the honeybee combs cells. The stored pollen is mixed with the digestive enzymes secreted by bees, honey, and organic acids. Furthermore, under anaerobic conditions, BP is converted to BB by lactic acid fermentation caused by bacteria (Pseudomonas spp. and Lactobacillus spp.) and yeasts (Saccharomyces spp.) (Detry, Simon-Delso, Bruneau, \& Daniel, 2020; Di Cagno, Filannino, Cantatore, \& Gobbetti, 2019; Mărgăoan, Cornea-Cipcigan, Topal, \& Kösoğlu, 2020; Tomás et al., 2017), (Fig. 1). As the BP turns into BB, it goes through a number of biochemical stages which is completed in about 7 days: i) growing microorganisms such as lactic acid bacteria, Escherichia, aerobic bacteria and yeasts in the first half day, ii) using nutrient by bacteria and drop in $\mathrm{pH}$, iii) loss of Streptococcus bacteria and growth of Lactobacillus bacteria, and iv) death of lactic acid bacteria and some yeasts due to the produced lactic acid (Khalifa et al., 2020).

BB contains protein and amino acids, carbohydrates, lipids, vitamins, minerals and phenolic acids and polyphenols (Tomás et al., 2017; Zuluaga et al., 2015b), similar to BP, but with a higher nutrient-rich content (Adaškevičiūtè, Kaškoniene, Kaškonas, Barčauskaitè, \& Maruška, 2019). Another important point is the BB higher digestibility and degree of absorption by humans since the multi-layered wall surrounding the pollen grain is destroyed by natural fermentation, which gives special features to the BB (Khalifa et al., 2020; Kieliszek et al., 2018; Mizrahi \& Lensky, 2013). Recent studies have reported that BP and BB have anti-inflammatory, anti-obesity, anticancer, antimicrobial, antioxidant and gastroprotective properties as well as neuroprotective

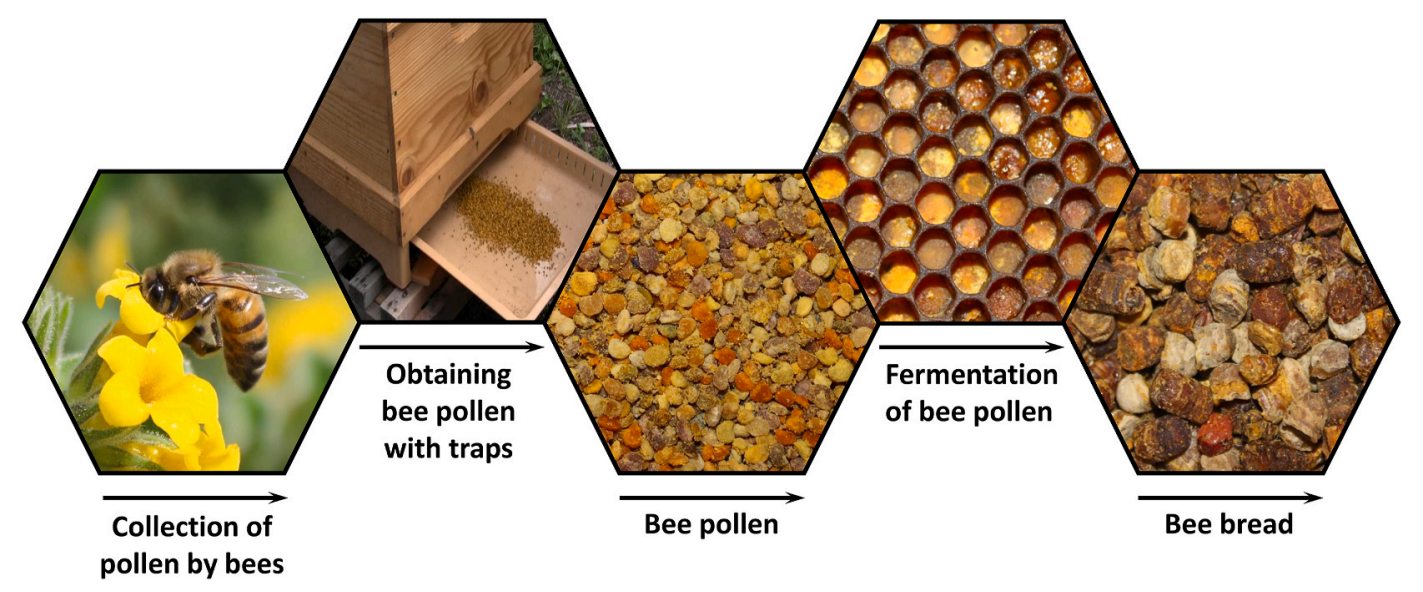

Fig. 1. Pollen chain: from collection of pollen by bees to the formation of bee bread. 
and anti-aging activity (Bacha et al., 2020; Bakour et al., 2019; Eleazu et al., 2020; Kurek-Górecka, Górecki, Rzepecka-Stojko, Balwierz, \& Stojko, 2020; Othman et al., 2020; Selamoglu, 2017; Sobral et al., 2017; Tomás et al., 2017).

\section{Differences between bee pollen and bee bread}

BP and BB have similar composition due to their similar origin of plant pollen. Albeit their similarity, there are remarked differences, Table 1. The breakdown of the multi-layered wall of BP during fermentation and the formation of $\mathrm{BB}$ make it more digestible than $\mathrm{BP}$ (Khalifa et al., 2020). This process increases the bioavailability of BB and results in a higher degree of absorption by human intestinal epithelial cells (Zuluaga, Serrato, \& Quicazan, 2015). Cellulose, which forms the inner layer (intine) of $\mathrm{BP}$, is broken down by bacteria, reducing the cellulose ratio of BB compared to BP (Benavides-Guevara, Quicazan, \& Ramírez-Toro, 2017). Furthermore, during this transformation process some new products are formed. For example, some of the proteins in BP are reduced to amino acids by digestive enzymes, so the protein content in BP is higher, while in BB a higher amount of amino acids can be found (Kieliszek et al., 2018). The study conducted by DeGrandi-Hoffman, Eckholm, and Huang (2013) showed that the concentration of leucine and threonine in BB is about $60 \%$ higher than in BP. In the same study, it was emphasized that other amino acids such as valine, alanine, aspartic acid, and glutamine have a higher concentration in the BB. Differences in sugar content can also be found: BP is mixed with honey when stored in comb cells, as a result BB is richer in sugar than BP (Khalifa et al., 2020; Thakur \& Nanda, 2020).

The fermentation process of BB leads to a high content of lactic acid as a result of the metabolism of microorganisms, which will give a long time protection against microorganisms, as well as contributing to the nutritional properties of BB (Khalifa et al., 2020; Mayda et al., 2020). However, lactic acid decreases the level of $\mathrm{pH}$. While the $\mathrm{pH}$ of $\mathrm{BP}$ varies between 4 and 6, the $\mathrm{pH}$ of $\mathrm{BB}$ is around 4.2 (Khalifa et al., 2020; Kieliszek et al., 2018). Moreover, BB has a richer content in vitamins than $\mathrm{BP}$, for example, BB has a higher content in terms of vitamin $\mathrm{K}$ (Khalifa et al., 2020) which may result from pollen degradation. Concerning fatty acids, BP and BB have a similar content (Kieliszek et al., 2018).

Table 1

Composition of bee pollen and bee bread.

\begin{tabular}{|c|c|c|c|}
\hline Composition & Bee pollen & $\begin{array}{l}\text { Bee } \\
\text { bread }\end{array}$ & References \\
\hline Carbohydrates & $13-55 \%$ & $24-35 \%$ & $\begin{array}{l}\text { Campos et al. (2010); Khalifa et al. } \\
\text { (2020) }\end{array}$ \\
\hline Proteins & $10-40 \%$ & $14-23 \%$ & \begin{tabular}{l} 
Campos et al. (2010); Tomás et al. \\
(2017); Zuluaga et al. (2015a)\} \\
\hline Lipids & $1-13 \%$ & $2-14 \%$ & $\begin{array}{l}\text { Bakour et al. (2019); Campos et al. } \\
\text { (2010); De-Melo, Estevinho, } \\
\text { Moreira, Delerue-Matos, Freitas, } \\
\text { et al. (2018); Tomás et al. (2017) }\end{array}$ \\
\hline Dietary fiber & $14-31 \%$ & $3-20 \%$ & $\begin{array}{l}\text { Anjos, Paula, Delgado, and Esrevinho } \\
\text { (2019); Salazar-González and } \\
\text { Díaz-Moreno (2016); K. Yang et al. } \\
\text { (2013) }\end{array}$ \\
\hline Vitamins & $0.02-0.7 \%$ & $0.4-3 \%$ & $\begin{array}{l}\text { Farag and El-Rayes (2016); Urcan } \\
\text { et al. (2018) }\end{array}$ \\
\hline Amino acids & $3.2 \%$ & n.i. & K. Yang et al. (2013) \\
\hline Organic acids & $1 \%$ & 0.4 & $\begin{array}{l}\text { Bakour et al. (2019); Moita et al. } \\
\text { (2014) }\end{array}$ \\
\hline Flavonoids & $0.2-3.2 \%$ & n.i. & Bogdanov (2011) \\
\hline Lactic acid & $0.6 \%$ & $3 \%$ & Kieliszek et al. (2018) \\
\hline $\begin{array}{r}\text { Free acidity } \\
(\mathrm{mEq} / \mathrm{kg})\end{array}$ & $105-146$ & 400 & $\begin{array}{l}\text { Bakour et al. (2019); Bárbara et al. } \\
\text { (2015); Martins, Morgano, Vicente, } \\
\text { Baggio, and Rodriguez-Amaya } \\
\text { (2011) }\end{array}$ \\
\hline $\mathrm{pH}$ & $4-6.3$ & $3.8-4.2$ & $\begin{array}{l}\text { Khalifa et al. (2020); Kieliszek et al. } \\
\text { (2018) }\end{array}$ \\
\hline
\end{tabular}
\end{tabular}

n.i.: no information.
The process of production from the hive is also distinct and clearly more difficult in BB than BP. The placed traps at the entrance of the hives are often sufficient to obtain BP. But BB is tightly packed and fixed with beeswax in combs by bees, therefore more laborious techniques are required to obtain it, such as manual crushing of the combs after freezing or machinery separation. As a result, $\mathrm{BB}$ has a higher price commercially than other bee products such as BP, honey, or beeswax.

\section{Plant origin}

The information of BP and BB botanical origin is crucial because it can work as an indicator of nutritional values, phytochemicals, biological activity and may influence its commercial quality (Campos et al., 2010). The pollen collected by bees differ in color, size, appearance, biological activity and physicochemical features (Čeksteryte et al., 2016). This difference is due to geographical, seasonal conditions and genetics of flower species that are visited by bees, which have unique characteristics (De-Melo, Estevinho, Moreira, Delerue-Matos, Freitas, et al., 2018). Also, morphological aspects such as the pore structure and the thickness of the multiple layers of the pollen grains depend on botanical source.

BP is called monofloral when only one single botanical taxonomy characterizes the pollen loads while preserving the physical and biochemical properties of the plant, whereas mix-pollen loads from various flowers are called polyfloral (Carpes et al., 2013). At commercial level, BP and BB are mostly sold as polyfloral, as a result of the mixture of pollen pellets collected in the traps or storage by the bees in the same honeycomb.

The traditional approach to access the botanical origin of both pollen products is based on microscopic analysis, which is time-consuming and requires good palynology analysis expertise. With advances in molecular biology and new analytical methods, new approaches are under development. One of these is based on the use of free amino acids, minerals, and aroma compounds, but it introduces both complex and expensive materials (Laha et al., 2017). DNA barcoding technology and next-generation sequencing of amplicons seems more promising (Laha et al., 2017) with higher sensitivity and resolution than microscopic analysis. However, some limitations remain due to the incomplete plant database, access to laboratory equipment, and evaluation of mixed pollen samples originating from more than one plant species (Keller et al., 2015).

\section{Structure and digestibility of the pollen grain}

BP grains are surrounded by a strong outer layer called exine and an inner layer called intine, both protecting the pollen grains against physical and chemical agents. The exine layer consists of chemically inert biopolymer sporopollenin and has a flexible and solid structure, while the intine layer consists of cellulose and pectin and has a more sensitive structure compared to the exine layer (Fig. 2) (Benavides-Guevara et al., 2017; F.-S.; Li, Phyo, Jacobowitz, Hong, \& Weng, 2019; Zuluaga et al., 2015b). This multi-layered structure ensures that the pollen grains are resistant to bacteria, and also preserve the pollen content against environmental factors like temperature, $\mathrm{pH}$-changes, or ultraviolet radiation (Zuluaga-Domínguez, Serrato-Bermudez, \& Quicazán, 2018). Indeed, this protection is also effective against human digestion: the absence of specific enzymes, in the human gastrointestinal tract (GIT), for the digestion of the multiple layers surrounding the pollen grains reduces the effectiveness of the digestion (Benavides-Guevara et al., 2017; Rzepecka-Stojko, Stojko, Kurek-Górecka, Górecki, Kabała-Dzik et al., 2015). Differently, BB is more digestible by humans than BP because it is subject to the fermentation process in the combs (Khalifa et al., 2020). The botanical origin may also affect the digestibility degree of bee products (Thakur \& Nanda, 2020). Pollen grains with more and larger pores may be easier and faster to digest because digestive enzymes can penetrate easier into the cytoplasmic 


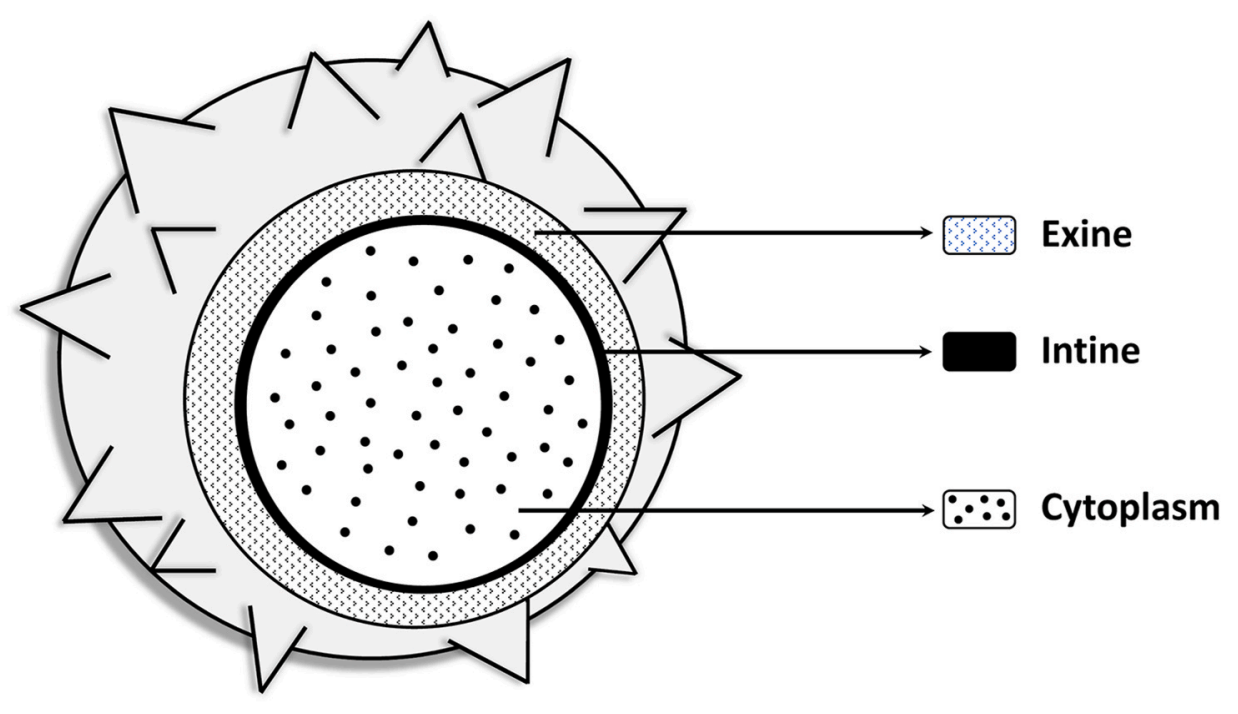

Fig. 2. Cross-section through a pollen grain and its multi-layered structure.

content, resulting in the more effective release of the inner content of pollen grains. On the other hand, pollen with thinner layers is destroyed more effectively by fermentation bacteria, consequently, their bioaccessibility degree may also be higher (Benavides-Guevara et al., 2017; Di Cagno et al., 2019).

Several studies have reported that fermentation is an effective method for the destruction of the pollen layers in order to reach the rich cytoplasmic content of pollen grains, however, this mechanism has not been entirely revealed yet. For instance, Z. Zhang et al. (2017) used fermentation through Ganoderma lucidum and Saccharomyces cerevisiae in order to break the BP layer and reported a yield of about $85 \%$ for G. lucidum and $88 \%$ for $S$. cerevisiae. In another study (Kaškonienè, Adaškevičiūte, Kaškonas, Mickiene, \& Maruška, 2020), the antibacterial, antifungal and antioxidant activities of BP were evaluated before and after fermentation with Lactococcus lactis and Lactobacillus rhamnosus bacteria. According to the obtained results, the total phenolic and flavonoid content increased significantly after the fermentation process and thus, an increase in antioxidant, antibacterial and antifungal activity, was observed. In addition, different methods have been proposed to increase the digestibility and consequently bioavailability of BP grains, such as the mechanical breakdown of the pollen layer, thermal shocks, penetration with digestive enzymes, or the disintegration of the pollen layers with osmotic shock (T'ai \& Cane, 2000).

Breaking the layers of BP may increase digestibility, but it should be considered that the use of thermal shocks or chemical agents may negatively affect phytochemical compounds in BP (Clement, Olatunde, Obigwa, \& Orijajogun, 2017), besides, without layers BP will be more sensitive to harmful environmental factors and microbial contaminations, which may limit the shelf time of the product.

\section{Content of dietary phytochemicals in bee pollen and bee bread}

Today, botanical resources are explored as dietary sources rich in macro and micro nutrients and particularly phytochemicals (Bakour et al., 2019; Gardana et al., 2018; Khalifa et al., 2020). Phytochemicals are plant secondary metabolites which accumulate in different structures, especially in the skin part of the plant that may play little or no role in plant metabolism (Crozier, Del Rio, \& Clifford, 2010). In BP and $\mathrm{BB}$, phytochemicals are very diverse and strongly dependent on the botanical origin of the pollen visited and collected by bees (Carpes et al., 2013), including phenolic acids, flavonoids, carotenoids or vitamins.

\subsection{Phenolic compounds}

Phenolic compounds have a chemical structure that includes aromatic ring(s) carrying one or more hydroxyl groups. Over 8000 phenolic compounds, which are spread over a wide range in the plant world, have been identified, and may be classified differently according to the number of carbon atoms or structural elements between the phenol rings (Tsao, 2010). In addition, these may be found in different structures, from low molecular weight to complex high molecular weight phenolic derivatives. Phenolic compounds are basically divided into two main groups: flavonoids and phenolic acids (Fig. 3). Flavonoids are the most common compounds in the plant-derived diet (Crozier, Jaganath, \& Clifford, 2009; Tsao, 2010). They are chemically attached to two benzene rings by a three-carbon bridge with a skeleton containing 15 carbons (C6-C3-C6). Sugars, like glucose, rhamnose or arabinose, or organic acids (e.g. glucuronic acid) are generally attached to flavonoids (Stahl et al., 2002). Depending on attached hydroxyl groups, position and saturation level, dietary flavonoids can be sub classified as flavonols, flavones, flavan-3-ols, anthocyanidins, flavanones and isoflavones. Phenolic acids (C6-C1) are derivatives of hydroxycinnamic or hydroxybenzoic acids. The most common hydroxycinnamic acids are caffeic acid, ferulic acid, $p$-coumaric acid and synaptic acid, while benzoic acid, syringic acid, vanillic acid and gallic acid are the most common hydroxybenzoic acid derivatives (Abbas et al., 2017; Crozier et al., 2009).

BP and BB are characterized by a rich phenolic profile which is reflected on the therapeutic and biological properties (Araújo et al., 2017; Baltrušaitytè, Venskutonis, \& Čeksterytè, 2007; Denisow \& Denisow-Pietrzyk, 2016). The total phenolic content (TPC) and total flavonoid content (TFC) of these bee products are on one hand strongly dependent on the botanical origin of the pollen (Gardana et al., 2018), but also on the solvent used in the extraction methodology applied for their determination, Table 2. Observed values for TPC and TFC of BP range between 0.7 and $136 \mathrm{mg} \mathrm{GAE} / \mathrm{g}$ and $0.1-78 \mathrm{mg} \mathrm{QE} / \mathrm{g}$, respectively, while for $\mathrm{BB}$, those values vary between 4.4 and $84 \mathrm{mg} \mathrm{GAE} / \mathrm{g}$ and 1.7-96 mg QE/g, respectively. Besides, considering the reported studies, BP and BB samples with high TFC and TPC exhibit higher antioxidant activity (Baltrušaityte et al., 2007).

As previously specified, flavonoids are the major group of phenolic compounds found in BP and BB (Rzepecka-Stojko, Stojko, Kurek-Górecka, Górecki, Kabała-Dzik et al., 2015). Table 3 list the richness of those phytochemical found in BP and BB across the world with a clear dominance of flavonols, particularly the derivatives of kaempferol, quercetin, isorhamnetin and myricetin (Anjos, Fernandes, et al., 2019; 


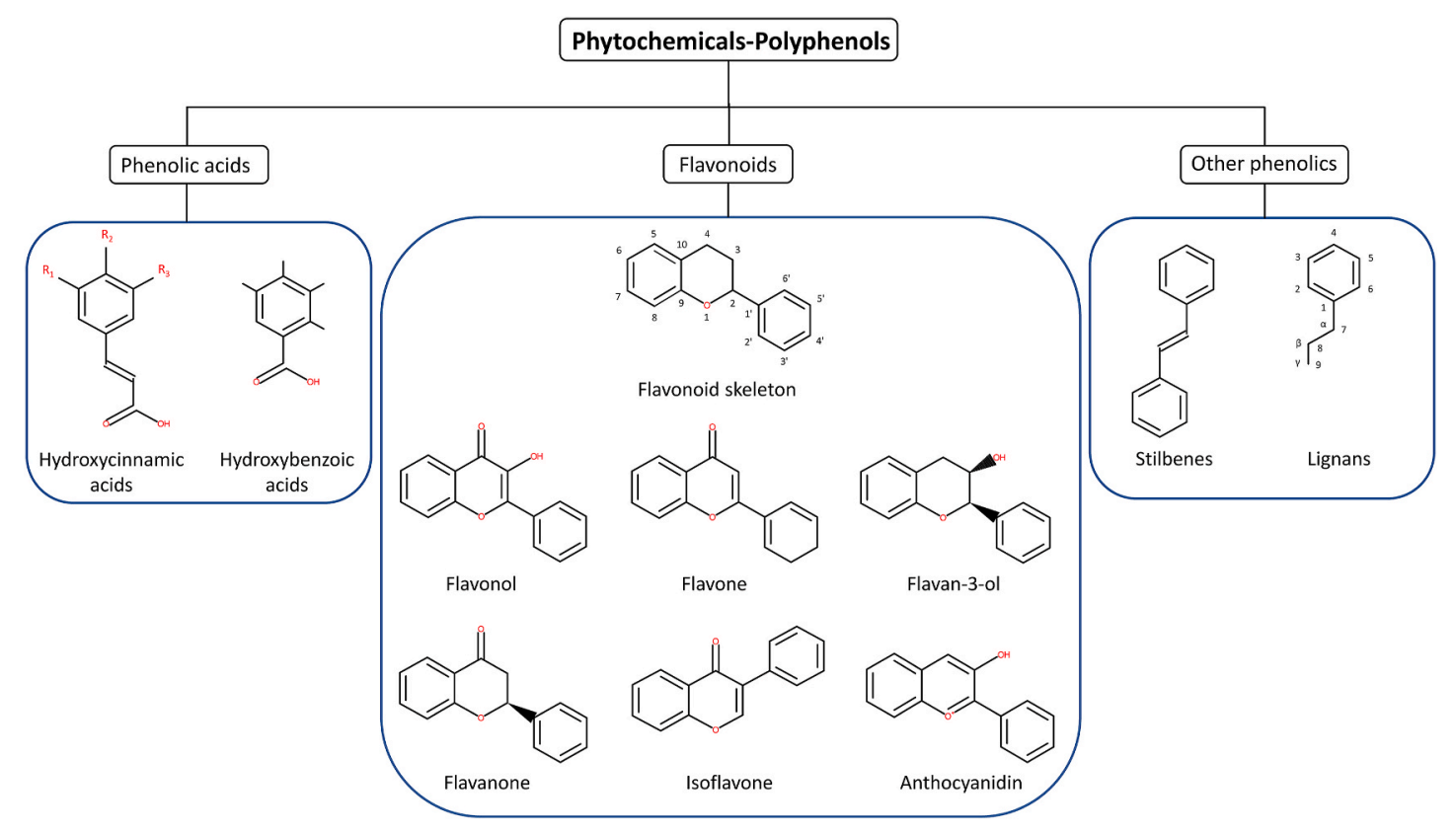

Fig. 3. Classification and chemical structure of some dietary phytochemicals present in bee pollen and bee bread.

Bakour et al., 2019; Čeksteryte et al., 2016; Karabagias, Karabagias, Gatzias, \& Riganakos, 2018; Kostić et al., 2019; Negri, Barreto, Sper, Carvalho, \& Campos, 2018; Othman et al., 2020; Sobral et al., 2017; Urcan et al., 2018). Flavones are also frequently present, particularly with derivatives of apigenin and luteolin.

Dietary bioactive flavonoids present in BP and BB have different pharmacological and medical roles such as antioxidant activity, enzyme activation, gene expression, hormone regulation, anti-inflammation, neuroprotective, anticancer and antidiabetic (Abouda, Zerdani, Kalalou, Faid, \& Ahami, 2011; Bacha et al., 2020; Denisow \& Denisow-Pietrzyk, 2016; Kieliszek et al., 2018; Othman et al., 2020), but its effectiveness to prevent diseases, is highly dependent on the degree of bioavailability and assimilation by humans (Carbonell-Capella, Buniowska, Barba, Esteve, \& Frígola, 2014; Crozier et al., 2010). Choi, Tai, Cuong, Kim, and Jang (2012) studied the anti-oxidative and anti-inflammatory effects of dietary quercetin and its glycosides as in vitro, with quercetin showing poor antioxidant activity compared to its glycosides. On the other hand, quercetin was higher in inducible nitric oxide synthesis and nuclear factor kappa B activation than its glycosides which may be associated with higher anti-inflammatory activity. In addition, other reports have shown that quercetin has anti-carcinogenic activity on different cancer cell lines and tumors (Dajas, 2012). Also, Zang, Zhang, Igarashi, and Yu (2015) investigated the anti-obesity and anti-diabetic effects of kaempferol and glycosides in mice, and they reported that kaempferol glycosides have the potential to reduce the aggregation of adipose tissue, hyperlipidemia and diabetes by enhancing lipid metabolism.

Isorhamnetin, another dominant flavonoid in BP and BB, a methylated form of quercetin, is known to have activities such as anti-cancer, anti-proliferative and anti-inflammation (Hämäläinen, Nieminen, Vuorela, Heinonen, \& Moilanen, 2007). Besides that, stimulation of phase II antioxidant enzyme expression and protective properties against oxidative damage of isorhamnetin was examined by J. H. Yang et al. (2014). They concluded that isorhamnetin induces the expression of phase II antioxidant enzyme and is effective in protecting hepatocytes against oxidative stress by preventing the accumulation of reactive oxygen species (ROS) with the activation of NF-E2-related factor-2. Given the above, it is clear that the active diet phytochemicals in $\mathrm{BP}$ and $\mathrm{BB}$ may play a key role in the prevention, treatment and management of different diseases.

\subsection{Carotenoids}

Carotenoids are a group of pigmented compounds consisting of a single carbon chain synthesized by plants and some microorganisms (e. g., bacteria and algae). Carotenoids protect plants against oxidative stress and photo-damage (Abd Alla \& Salem, 2020). Approximately 100 dietary carotenoids are included in people's diet within coloring foodstuffs (Bohoyo-Gil, Dominguez-Valhondo, Garcia-Parra, \& González-Gómez, 2012). BP and BB are rich sources of dietary carotenoids, which are responsible for its different colors, such as yellow, orange, brown and many others, which vary according to its botanical origin (Thakur \& Nanda, 2020). $\beta$-cryptoxanthin, lutein and lycopene are the most common types of carotenoids found in these bee products (Mărgă;oan et al., 2014), Table 4. The amount of carotenoids found for BP samples in literature is set in the range between 5 and $1233.0 \mu \mathrm{g} / \mathrm{g}$ (Sattler et al., 2015), nevertheless, there are no results reported regarding the quantitative analysis on carotenoid content of BB.

Carotenoids are known to play a key role in cell growth, regulation of dysfunction on a cellular basis, and preventing common diseases such as cancer (Ross, 2010). Previously, Y. Zhang et al. (2016) evaluated in vitro and in vivo the use of $\beta$-carotene with 5 -fluorouracil (a used drug in cancer treatment) in the treatment of human esophageal cancer and reported that its combined use increased the inhibition activity on esophageal cancer cells compared to individual use of them. Carotenoids have also strong antioxidant activity, due to the presence of the conjugated double bond system in their structure. They are known as scavengers of ROS and contribute greatly to the prevention of many common chronic diseases (Ross, 2010).

\subsection{Vitamins}

Vitamins are a group of organic compounds with diverse biochemical roles and strong antioxidant activity. Although vitamins are included in the human diet in small amounts, they are vital micro compounds for the development and maintenance of health (Gey, 1998). Vitamins are naturally found in foods, especially vegetables and fruits, or plant-derived foods, and play a role in managing and regulating metabolic and cellular energy processes (Gey, 1998). The beneficial effects on health are correlated with their optimal diary uptake. The shortage and excess of vitamins disrupt some metabolic processes and consequently, 
Table 2

Summary of total phenolic content (TPC) and total flavonoid content (TFC) of bee pollen and bee bread from different botanical origins throughout worldwide.

\begin{tabular}{|c|c|c|c|c|c|c|c|}
\hline \multirow{2}{*}{$\begin{array}{l}\text { Bee } \\
\text { product }\end{array}$} & \multirow[t]{2}{*}{ Country } & \multirow[t]{2}{*}{ Bee specie } & \multirow[t]{2}{*}{ Botanical source } & \multirow[t]{2}{*}{ Extraction solvent } & \multicolumn{2}{|c|}{ Range quantification } & \multirow[t]{2}{*}{ References } \\
\hline & & & & & $\begin{array}{l}\text { Total phenolic } \\
\text { content (TPC) }\end{array}$ & $\begin{array}{l}\text { Total flavonoid } \\
\text { content (TFC) }\end{array}$ & \\
\hline \multirow[t]{13}{*}{ BP } & Brazil & Apis mellifera & $\begin{array}{l}\text { M. caesalpiniifolia, Cocos } \\
\text { nucifera, Mimoza scabrella, } \\
\text { Okaliptïs, Asteraceae, } \\
\text { Eupatoryum, Coffea, Myrcia, } \\
\text { Poaceae, Rubiaceae, Cecropia, } \\
\text { A. aculeatissimum, Astronyum, } \\
\text { Mimoza verrucosa, Fabaceae, } \\
\text { Myrtaceae, M. scabrella, } \\
\text { Richardia, Piper, Elephantopus, } \\
\text { Syagrus, Vernonia, Ilex, } \\
\text { Schinus, Arecaceae, } \\
\text { Anadenanthera, Malvaceae, } \\
\text { Alternanthera, Ricinus, } \\
\text { Montanoa, Machaerium, } \\
\text { Antigonon, Brassica, Apiaceae, } \\
\text { Baccharis, Anacardiaceae, } \\
\text { Loranthaceae, Sebastiania and } \\
\text { Melastomataceae }\end{array}$ & Ethanol & $\begin{array}{l}6.7-29.2 \mathrm{mg} \\
\mathrm{GAE} / \mathrm{g}\end{array}$ & $\begin{array}{l}0.9-17.5 \mathrm{mg} \\
\mathrm{QE} / \mathrm{g}\end{array}$ & $\begin{array}{l}\text { De-Melo, Estevinho, } \\
\text { Moreira, Delerue-Matos, } \\
\text { Freitas, et al. (2018) }\end{array}$ \\
\hline & USA & n.i. & $\begin{array}{l}\text { Mesquite, Yucca, Palm, } \\
\text { Terpentine Bush, Mimosa and } \\
\text { Chenopod }\end{array}$ & Methanol & $\begin{array}{l}15.91-34.85 \\
\mathrm{mg} \mathrm{GAE} / \mathrm{g}\end{array}$ & n.i. & $\begin{array}{l}\text { LeBlanc, Davis, Boue, } \\
\text { DeLucca, and Deeby } \\
\text { (2009) }\end{array}$ \\
\hline & Poland & n.i. & $\begin{array}{l}\text { Brassicaceae, Asteraceae, } \\
\text { Taraxacum, Centaurea cyanus, } \\
\text { Fagopyrum, Trifolium, } \\
\text { Apiaceae, Poaceae, Tilia, } \\
\text { Rosaceae and Aesculus }\end{array}$ & $\begin{array}{l}\text { Water, ethanol and } \\
\text { methanol }\end{array}$ & $\begin{array}{l}2.95-31.52 \mathrm{mg} \\
\mathrm{GAE} / \mathrm{g}\end{array}$ & n.i. & $\begin{array}{l}\text { Borycka, Grabek-Lejko, } \\
\text { and Kasprzyk (2015) }\end{array}$ \\
\hline & Portugal & n.i. & $\begin{array}{l}\text { Cistus ladanifer, Echium, } \\
\text { Apiaceae, Brassicaceae spp., } \\
\text { Cichorieae spp., Asteraceae } \\
\text { spp., Lavandula spp., Plantago } \\
\text { spp. and Silene spp. }\end{array}$ & Ethanol & $\begin{array}{l}35.05 \mathrm{mg} \mathrm{GAE} / \\
\mathrm{g}\end{array}$ & $6.99 \mathrm{mg} \mathrm{QE} / \mathrm{g}$ & $\begin{array}{l}\text { Anjos, Fernandes, et al. } \\
\text { (2019) }\end{array}$ \\
\hline & & n.i. & $\begin{array}{l}\text { Rubus spp., Castanea sativa, } \\
\text { Cistus spp., Leontondon spp., } \\
\text { Echium spp., Echium spp., } \\
\text { Eucalyptus spp., and Erica spp. }\end{array}$ & Methanol & $\begin{array}{l}14.83-43.97 \\
\mathrm{mg} \mathrm{GAE} / \mathrm{g}\end{array}$ & $\begin{array}{l}2.49-11.83 \mathrm{mg} \\
\mathrm{CAE} / \mathrm{g}\end{array}$ & Anjos, Paula, et al. (2019) \\
\hline & & Apis mellifera & $\begin{array}{l}\text { Cistus, Echium, Prunus, } \\
\text { Castanea, Leontodon, } \\
\text { Trifollium, Erica, Quercus, } \\
\text { Mimosa, Eucalyptus and Rubus }\end{array}$ & Methanol & $\begin{array}{l}12.90-19.80 \\
\mathrm{mg} \mathrm{GAE} / \mathrm{g}\end{array}$ & $\begin{array}{l}4.50-7.0 \mathrm{mg} \\
\mathrm{CAE} / \mathrm{g}\end{array}$ & $\begin{array}{l}\text { Feás, Vázquez-Tato, } \\
\text { Estevinho, Seijas, and } \\
\text { Iglesias (2012) }\end{array}$ \\
\hline & Brazil & Apis mellifera & $\begin{array}{l}\text { Cocos nucifera, Miconia spp., } \\
\text { Spondias spp., Myrcia spp., } \\
\text { Saccharum spp., Eucalyptus } \\
\text { spp., Mikania spp., and } \\
\text { Mimosa spp. }\end{array}$ & Methanol & $\begin{array}{l}33.73-75.60 \\
\mathrm{mg} \mathrm{GAE} / \mathrm{g}\end{array}$ & $\begin{array}{l}1.42-9.05 \mathrm{mg} \\
\mathrm{QE} / \mathrm{g}\end{array}$ & Araújo et al. (2017) \\
\hline & & Apis mellifera & $\begin{array}{l}\text { Brassicaceae, Asteraceae } \\
\text { Elephantopus, Asteraceae } \\
\text { Eupatorium, Asteraceae } \\
\text { Gochnatia, Myrtaceae } \\
\text { eucalyptus and Loranthaceae } \\
\text { Struthanthus }\end{array}$ & Ethanol & $\begin{array}{l}30.55-48.76 \\
\mathrm{mg} \mathrm{GAE} / \mathrm{g}\end{array}$ & $\begin{array}{l}8.55-28.43 \mathrm{mg} \\
\mathrm{QE} / \mathrm{g}\end{array}$ & Carpes et al. (2013) \\
\hline & Italy & n.i. & $\begin{array}{l}\text { Castanea sp., Rubus sp., and } \\
\text { Cistus sp., }\end{array}$ & Ethanol & $\begin{array}{l}13.53-24.75 \\
\mathrm{mg} \mathrm{GAE} / \mathrm{g}\end{array}$ & n.i. & Domenici et al. (2015) \\
\hline & Poland & n.i. & n.i. & Ethanol & $\begin{array}{l}13.24-27.03 \\
\mathrm{mg} \mathrm{GAE} / \mathrm{g}\end{array}$ & $\begin{array}{l}11.22-77.88 \\
\mathrm{mg} \mathrm{QE} / \mathrm{g}\end{array}$ & $\begin{array}{l}\text { Rzepecka-Stojko, Stojko, } \\
\text { Kurek-Górecka, Górecki, } \\
\text { Sobczak, et al. (2015) }\end{array}$ \\
\hline & $\begin{array}{l}\text { Spain and } \\
\text { Portugal }\end{array}$ & n.i. & $\begin{array}{l}\text { Cistaceae, Fabaceae, } \\
\text { Ericaceae and Boraginaceae }\end{array}$ & Methanol & $\begin{array}{l}\text { 18.55-32.15 } \\
\text { mg GAE/g }\end{array}$ & $\begin{array}{l}3.71-10.14 \mathrm{mg} \\
\mathrm{CAE} / \mathrm{g}\end{array}$ & $\begin{array}{l}\text { Pascoal, Rodrigues, } \\
\text { Teixeira, Feás, and } \\
\text { Estevinho (2014) }\end{array}$ \\
\hline & Romania & n.i. & $\begin{array}{l}\text { Brassica napus, Taraxacum } \\
\text { officinale, Malus domestica, } \\
\text { Rubus idaeus, Taraxacum } \\
\text { officinale and Rosa canina }\end{array}$ & Ethanol & $\begin{array}{l}\text { 10.08-20.48 } \\
\mathrm{mg} \mathrm{GAE} / \mathrm{g}\end{array}$ & n.i. & $\begin{array}{l}\text { Stanciu, Marghitas, and } \\
\text { Dezmirean (2008) }\end{array}$ \\
\hline & Turkey & n.i. & $\begin{array}{l}\text { Chenopodiaceae, Cistus sp., } \\
\text { Convolvulus sp., Scabiosa sp., } \\
\text { Rhododendron sp., Euphorbia } \\
\text { sp., Astragalus sp., Coronilla } \\
\text { sp., Hedysarum sp., Lotus sp., } \\
\text { Melilotus sp., Onobrychis sp., } \\
\text { Trifolium sp., Vicia sp., } \\
\text { Castanea sativa, Geraniaceae, } \\
\text { Hypericaceae, Lamiaceae } \\
\text { Salvia sp., Teucrium sp., }\end{array}$ & Ethanol & $\begin{array}{l}26.69-43.42 \\
\mathrm{mg} \mathrm{GAE} / \mathrm{g}\end{array}$ & $\begin{array}{l}2.62-4.44 \mathrm{mg} \\
\mathrm{QE} / \mathrm{g}\end{array}$ & Mayda et al. (2020) \\
\hline
\end{tabular}


Table 2 (continued)

\begin{tabular}{|c|c|c|c|c|c|c|c|}
\hline \multirow{2}{*}{$\begin{array}{l}\text { Bee } \\
\text { product }\end{array}$} & \multirow[t]{2}{*}{ Country } & \multirow[t]{2}{*}{ Bee specie } & \multirow[t]{2}{*}{ Botanical source } & \multirow[t]{2}{*}{ Extraction solvent } & \multicolumn{2}{|c|}{ Range quantification } & \multirow[t]{2}{*}{ References } \\
\hline & & & & & $\begin{array}{l}\text { Total phenolic } \\
\text { content (TPC) }\end{array}$ & $\begin{array}{l}\text { Total flavonoid } \\
\text { content (TFC) }\end{array}$ & \\
\hline & & & $\begin{array}{l}\text { Thymus sp., Laurus sp., } \\
\text { Liliaceae, Loranthus sp., Tilia } \\
\text { sp., Eucalyptus sp., } \\
\text { Papaveraceae, } \\
\text { Plantaginaceae, Zea sp., } \\
\text { Rumex sp., Ranunculaceae, } \\
\text { Rosaceae, Salix sp., } \\
\text { Scrophulariaceae, Linaria sp., } \\
\text { and Solanaceae }\end{array}$ & & & & \\
\hline & $\begin{array}{l}\text { Denmark, Italy, } \\
\text { Switzerland, } \\
\text { Slovakia, Poland } \\
\text { Spain, Lithuania, } \\
\text { Ukraine and } \\
\text { Latvia }\end{array}$ & n.i. & n.i. & Methanol & $\begin{array}{l}33.14-55.04 \\
\mathrm{mg} \text { RUE/10 g }\end{array}$ & $\begin{array}{l}\text { 10.68-48.31 } \\
\mathrm{mg} \text { RUE/10 g }\end{array}$ & $\begin{array}{l}\text { Adaškevičiūtè et al. } \\
\text { (2019) }\end{array}$ \\
\hline & Lithuania & n.i. & $\begin{array}{l}\text { Brassica napus, Trifolium } \\
\text { repens, Carum carvi L., } \\
\text { Trifolium pratense, Fagopyrum } \\
\text { esculentum and Salix spp. }\end{array}$ & Methanol & $\begin{array}{l}23.30 \mathrm{mg} \mathrm{GAE} / \\
\mathrm{g}\end{array}$ & n.i. & Čeksteryte et al. (2016) \\
\hline & China & n.i. & n.i. & Ethanol & $\begin{array}{l}12.57 \mathrm{mg} \mathrm{GAE} / \\
\mathrm{g}\end{array}$ & $\begin{array}{l}22.89 \text { mg RUE/ } \\
\mathrm{g}\end{array}$ & $\begin{array}{l}\text { Sun, Guo, Zhang, and } \\
\text { Zhuang (2017) }\end{array}$ \\
\hline & Egypt & n.i. & Trifolium alexandrinum $\mathrm{L}$. & $\begin{array}{l}\text { Ethanol, ethyl } \\
\text { acetate, } \\
\text { dichloromethane } \\
\text { and petrolium ether }\end{array}$ & $\begin{array}{l}0.8-2.3 \mathrm{mg} \\
\mathrm{GAE} / \mathrm{g}\end{array}$ & $\begin{array}{l}0.1-0.85 \mathrm{mg} \\
\mathrm{QE} / \mathrm{g}\end{array}$ & $\begin{array}{l}\text { AbdElsalam, Foda, } \\
\text { Abdel-Aziz, and Abd } \\
\text { (2018) }\end{array}$ \\
\hline & Greece & n.i. & $\begin{array}{l}\text { Papaver rhoes, Chamomila } \\
\text { recutita, Sinapis arvensis, Cistus } \\
\text { sp., Trifolium sp., Dorycnium } \\
\text { sp., Cichorium sp., Convolvulus } \\
\text { sp., Circium sp., Malva } \\
\text { sylvestris, Fumana sp., } \\
\text { Eucalyptus camaldulensis, } \\
\text { Anemone sp., Ononis sp., } \\
\text { Asphodelus sp. and Quercus ilex }\end{array}$ & Ethanol & $\begin{array}{l}15.20-60.20 \\
\mathrm{mg} \mathrm{GAE} / \mathrm{g}\end{array}$ & $\begin{array}{l}6.00-57.60 \mathrm{mg} \\
\mathrm{QE} / \mathrm{g}\end{array}$ & Karabagias et al. (2018) \\
\hline & Malaysia & $\begin{array}{l}\text { Stingless bee } \\
\text { (Trigona apicalis, } \\
\text { Trigona itama and } \\
\text { Trigona thoracica) }\end{array}$ & n.i. & Ethanol & $\begin{array}{l}33.46-135.93 \\
\mathrm{mg} \mathrm{GAE} / \mathrm{g}\end{array}$ & $\begin{array}{l}15.28-31.80 \\
\mathrm{mg} \mathrm{QE} / \mathrm{g}\end{array}$ & $\begin{array}{l}\text { Harif Fadzilah, Jaapar, } \\
\text { Jajuli, and Wan Omar } \\
\text { (2017) }\end{array}$ \\
\hline & India & n.i. & Brassica juncea & Ethanol & $\begin{array}{l}18.29 \mathrm{mg} \mathrm{GAE} / \\
\mathrm{g}\end{array}$ & n.i. & Ketkar et al. (2014) \\
\hline & Serbia & Apis mellifera & Helianthus annuus L. & $\begin{array}{l}\text { Methanol and } \\
\text { ethanol }\end{array}$ & $\begin{array}{l}2.91-3.82 \mathrm{mg} \\
\mathrm{GAE} / \mathrm{g}\end{array}$ & $\begin{array}{l}0.84-0.87 \mathrm{mg} \\
\mathrm{QE} / \mathrm{g}\end{array}$ & Kostić et al. (2019) \\
\hline & Slovakia & n.i. & Helianthus annuus L. & Ethanol & $\begin{array}{l}0.69-0.80 \mathrm{mg} \\
\text { GAE/g }\end{array}$ & n.i. & $\begin{array}{l}\text { Fatrcová-Šramková, } \\
\text { Nôžková, Máriássyová, } \\
\text { and Kačániová (2016) }\end{array}$ \\
\hline & China & n.i. & Brassica campestris L. & Acetone & $\begin{array}{l}33.646 \mathrm{mg} \\
\mathrm{GAE} / \mathrm{g}\end{array}$ & n.i. & Yan et al. (2019) \\
\hline \multicolumn{8}{|l|}{ BB } \\
\hline & Morocco & n.i. & n.i. & Ethanol & $4.88 \mathrm{mg} \mathrm{GAE} / \mathrm{g}$ & $1.67 \mathrm{mg} \mathrm{QE} / \mathrm{g}$ & Bakour et al. (2017) \\
\hline & Poland & n.i. & n.i. & Ethanol & $\begin{array}{l}6.15-27.80 \mathrm{mg} \\
\mathrm{GAE} / \mathrm{g}\end{array}$ & n.i. & $\begin{array}{l}\text { Kowalski and Lukasiewicz } \\
\text { (2017) }\end{array}$ \\
\hline & & n.i. & $\begin{array}{l}\text { Brassicaceae, Asteraceae, } \\
\text { Taraxacum, Centaurea cyanus, } \\
\text { Fagopyrum, Trifolium, } \\
\text { Apiaceae, Poaceae, Tilia, } \\
\text { Rosaceae and Aesculus }\end{array}$ & $\begin{array}{l}\text { Water, ethanol and } \\
\text { methanol }\end{array}$ & $\begin{array}{l}4.37-9.59 \mathrm{mg} \\
\text { GAE/g }\end{array}$ & n.i. & Borycka et al. (2015) \\
\hline & Romania & n.i. & n.i. & $\begin{array}{l}\text { Methanol, ethanol, } \\
\text { water }\end{array}$ & $\begin{array}{l}8.32-22.72 \mathrm{mg} \\
\mathrm{GAE} / \mathrm{g}\end{array}$ & n.i. & $\begin{array}{l}\text { Stanciu, Marghitas, and } \\
\text { Dezmirean (2007) }\end{array}$ \\
\hline & Ukraine & n.i. & n.i. & Ethanol & $\begin{array}{l}12.36-25.44 \\
\mathrm{mg} \mathrm{GAE} / \mathrm{g}\end{array}$ & $\begin{array}{l}13.56-18.24 \mu g \\
Q E / g\end{array}$ & Ivanišová et al. (2015) \\
\hline & Poland & n.i. & n.i. & Ethanol & $\begin{array}{l}33.43-36.52 \\
\mathrm{mg} \mathrm{GAE} / \mathrm{g}\end{array}$ & n.i. & $\begin{array}{l}\text { Markiewicz-Żukowska } \\
\text { et al. (2013) }\end{array}$ \\
\hline & Colombia & Apis mellifera & n.i. & Ethanol & $8.9 \mathrm{mg} \mathrm{GAE} / \mathrm{g}$ & $3.2 \mathrm{mg} \mathrm{QE} / \mathrm{g}$ & Zuluaga et al., (2015a) \\
\hline & Portugal & $\begin{array}{l}\text { Apis mellifera } \\
\text { iberiensis }\end{array}$ & $\begin{array}{l}\text { Castanea sativa, Plantago sp., } \\
\text { Rubus sp., T. Cytisus striatus, } \\
\text { Hedera helix, T. Solanum nigra, } \\
\text { Plantago sp., T. Ulex europaeus, } \\
\text { Erica sp., Lavandula sp., Salix } \\
\text { sp., Crataegus monogyna, } \\
\text { Echium sp., T. Anthemis } \\
\text { arvensis, Brassica sp., T. } \\
\text { Raphanus raphanistrum, } \\
\text { Sesamoides sp., Jasione }\end{array}$ & Ethanol & $\begin{array}{l}14-84 \mathrm{mg} \\
\mathrm{GAE} / \mathrm{g}\end{array}$ & $11-96 \mathrm{mg} \mathrm{QE} / \mathrm{g}$ & Tomás et al. (2017) \\
\hline
\end{tabular}


Table 2 (continued)

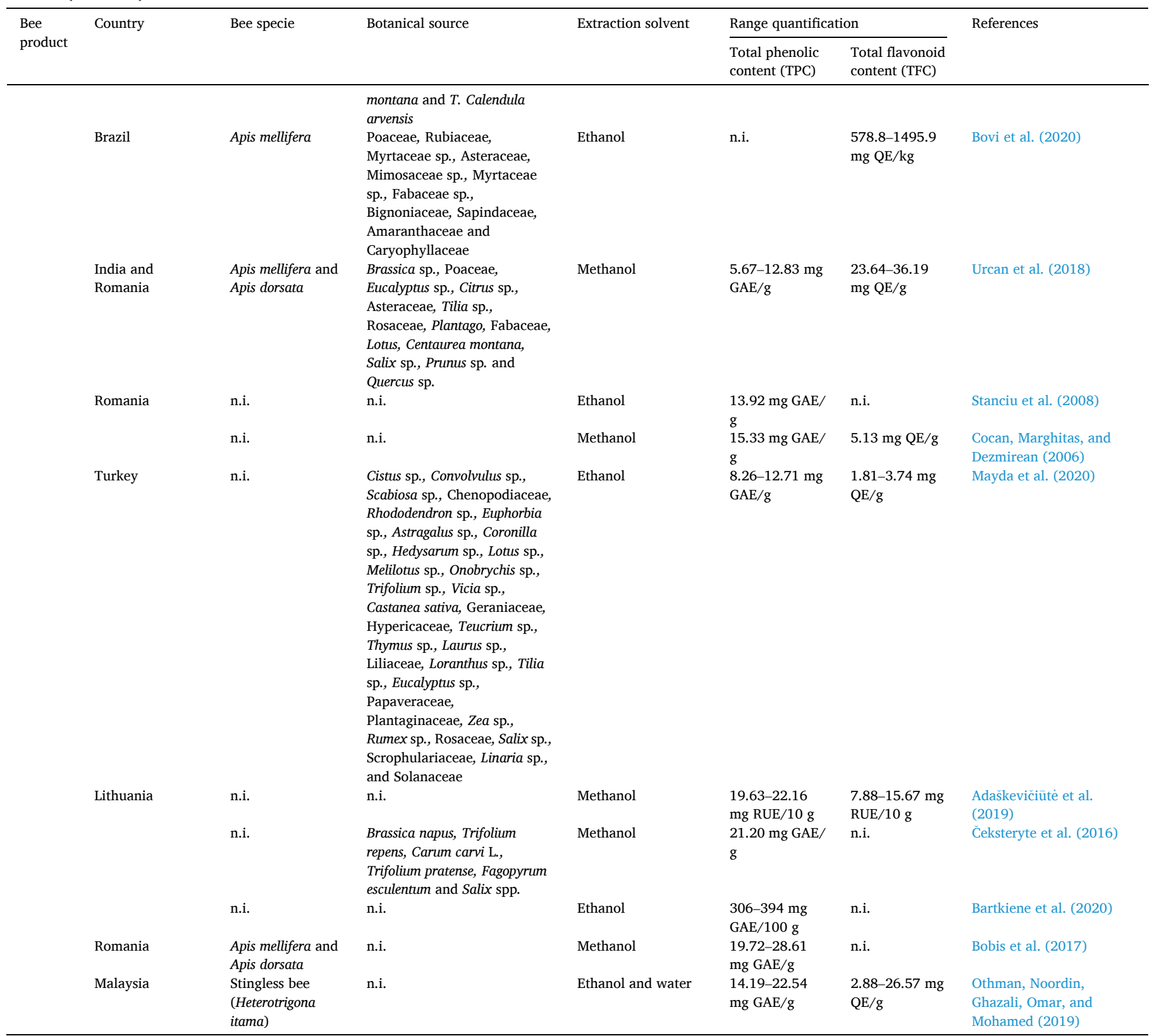

n.i., no information; GAE, gallic acid equivalent; QE quercetin equivalent; RUE, rutin equivalent; CAE, catechin equivalent.

different diseases can occur (Rizvi, Raza, Faizal Ahmed, Abbas, \& Mahdi, 2014). Therefore, an adequate intake of all vitamins is necessary for the functions of cells and tissues. Many studies have demonstrated that $\mathrm{BP}$ and $\mathrm{BB}$ are rich in vitamins, with an ideal vitamin content that may fulfill this necessity (Melo \& Almeida-Muradian, 2010; Oliveira et al., 2009; Tomás et al., 2017).

Both of them contain fat-soluble vitamins (A, D, E, and K) and watersoluble vitamins ( $\mathrm{C}$ and $\mathrm{B}$ complex), as well as vitamins such as vitamin P (Khalifa et al., 2020; Thakur \& Nanda, 2020), depending its contents on the botanical origin and collection season of the polen by the bees, as already mentioned. Both bee products contain vitamin E (Khalifa et al., 2020; Oliveira et al., 2009; Sattler et al., 2015), which is fat-soluble and is considered an antioxidant vitamin. Vitamin E can be stored in the body, so no daily intake is needed. Furthermore, it has many biological activities, including neuroprotective, anticancer, immune-enhancing and anti-inflammatory (Gey, 1998; Rizvi et al., 2014). The vitamin E family is collectively named tocochromanols, (tocopherols and tocotrienols). BP contains especially the tocopherols group ( $\alpha$-tocopherol, $\beta$-tocopherol, $\gamma$-tocopherol and $\delta$-tocopherol), being $\alpha$-tocopherol and $\gamma$-tocopherol the main compounds (Rizvi et al., 2014). Sattler et al. (2015) reported that $\alpha, \beta, \gamma$ and $\delta$-tocopherol contents of more than 20 samples of BP collected from different apiaries from southern Brazil presented 5-73 $\mu \mathrm{g} / \mathrm{g}, 1-10 \mu \mathrm{g} / \mathrm{g}, 2-12 \mu \mathrm{g} / \mathrm{g}$ and $1-84 \mu \mathrm{g} / \mathrm{g}$, respectively. In another study, the tocopherol content of BB from northern Portugal also set the higher value for $\delta$-tocopherol with ranges between 1 and 37 $\mathrm{mg} / \mathrm{g}$ for $\alpha$-tocopherol, $1-35 \mathrm{mg} / \mathrm{g}$ for $\beta+\gamma$-tocopherol and $77-293 \mathrm{mg} / \mathrm{g}$ for $\delta$-tocopherol (Tomás et al., 2017).

Vitamin C, another vitamin found in BP and BB, is a water-soluble vitamin and has a strong antioxidant property, which has been reported in different studies (Gey, 1998; Grosso et al., 2013; Sesso et al., 2008). BP content in vitamin C can vary between 14 and $797 \mu \mathrm{g} / \mathrm{g}$ (Melo \& Almeida-Muradian, 2010; Sattler et al., 2015), while in BB is reported to be much lower, around 0.06-0.11 mg/g (Haydak \& Palmer, 1942). Vitamin C plays a role as a co-factor in the synthesis of amino acids, 
Table 3

Phenolic compounds present in the bee pollen and bee bread.

\begin{tabular}{|c|c|c|c|c|c|}
\hline Phenolic compounds & Bee specie & Source & Extraction solvent & Spectral analysis & References \\
\hline \multicolumn{6}{|l|}{ Flavonols } \\
\hline \multirow[t]{2}{*}{ Quercetin } & Apis mellifera & BP & Methanol; ethanol & $\begin{array}{l}\text { UHPLC/MS-MS; } \\
\text { HPLC-PDA }\end{array}$ & $\begin{array}{l}\text { De-Melo, Estevinho, Moreira, } \\
\text { Delerue-Matos, Freitas, et al. (2018); Kostić } \\
\text { et al. (2019) }\end{array}$ \\
\hline & n.i. & $\mathrm{BB}$ & Methanol & LC-MS & Čeksteryte et al. (2016) \\
\hline Quercetin 3-O-galactoside & Apis mellifera & $\mathrm{BP}$ & Methanol; ethanol & UHPLC/MS-MS & Kostić et al. (2019) \\
\hline Quercetin-3-O-glucoside & Apis mellifera iberiensis & BB & Methanol & HPLC-DAD-ESI/MS & Sobral et al. (2017) \\
\hline \multirow[t]{2}{*}{ Quercetin 3-O-rhamnoside } & Apis mellifera & $\mathrm{BP}$ & Methanol; ethanol & UHPLC/MS-MS & Kostić et al. (2019) \\
\hline & Apis mellifera iberiensis & BB & Methanol & HPLC-DAD-ESI/MS & Sobral et al. (2017) \\
\hline \multirow[t]{2}{*}{ Quercetin 3-O-rutinoside (Rutin) } & Apis mellifera & $\mathrm{BP}$ & Methanol; ethanol & $\begin{array}{l}\text { UHPLC/MS-MS; } \\
\text { HPLC-PDA }\end{array}$ & $\begin{array}{l}\text { De-Melo, Estevinho, Moreira, } \\
\text { Delerue-Matos, Freitas, et al. (2018); Kostić } \\
\text { et al. (2019) }\end{array}$ \\
\hline & n.i. & BB & Methanol & LC-DAD-ESI/MS & Bakour et al. (2019) \\
\hline $\begin{array}{l}\text { Quercetin-3-O-rhamnosyl } \\
\text { glucoside }\end{array}$ & Apis mellifera & $\mathrm{BP}$ & $\begin{array}{l}\text { Dichloromethane; } \\
\text { methanol/water }\end{array}$ & $\begin{array}{l}\text { RP-HPLC-DAD-ESI- } \\
\text { MS/MS }\end{array}$ & Negri et al. (2018) \\
\hline Quercetin-O-dihexoside & n.i. & $\mathrm{BP}$ & Ethanol & UHPLC-DAD-ESI-MS & Anjos, Fernandes, et al. (2019) \\
\hline \multirow[t]{2}{*}{ Quercetin-3-O-sophoroside } & n.i. & $\mathrm{BP}$ & Methanol & LC-MS & Čeksteryte et al. (2016) \\
\hline & $\begin{array}{l}\text { Apis mellifera and Apis } \\
\text { dorsata }\end{array}$ & BB & Ethanol & HPLC/DAD & Urcan et al. (2018) \\
\hline \multirow[t]{2}{*}{ Quercetin-O-hexosyl-pentoside } & n.i. & $\mathrm{BP}$ & Ethanol & UHPLC-DAD-ESI-MS & Anjos, Fernandes, et al. (2019) \\
\hline & n.i. & $\mathrm{BB}$ & Methanol & LC-DAD-ESI/MS & Bakour et al. (2019) \\
\hline $\begin{array}{l}\text { Quercetin-3-O-glucosyl-6-O- } \\
\text { pentoside }\end{array}$ & Apis mellifera & $\mathrm{BP}$ & Ethanol & HPLC-MS & $\begin{array}{l}\text { De-Melo, Estevinho, Moreira, } \\
\text { Delerue-Matos, Freitas, et al. (2018) }\end{array}$ \\
\hline Quercetin-O-(malonyl)-rutinoside & n.i. & $\mathrm{BP}$ & Ethanol & UHPLC-DAD-ESI-MS & Anjos, Fernandes, et al. (2019) \\
\hline Quercetin-O-hexosyl-O-hexoside & n.i. & $\mathrm{BB}$ & Methanol & LC-DAD-ESI/MS & Bakour et al. (2019) \\
\hline Quercetin-O-hexosyl-O-rutinoside & Apis mellifera iberiensis & BB & Methanol & HPLC-DAD-ESI/MS & Sobral et al. (2017) \\
\hline Quercetin-O-(malonyl)-hexoside & n.i. & $\mathrm{BP}$ & Ethanol & UHPLC-DAD-ESI-MS & Anjos, Fernandes, et al. (2019) \\
\hline Bis-methylated quercetin & Apis mellifera & $\mathrm{BP}$ & Ethanol & HPLC-MS & $\begin{array}{l}\text { De-Melo, Estevinho, Moreira, } \\
\text { Delerue-Matos, Freitas, et al. (2018) }\end{array}$ \\
\hline \multirow[t]{2}{*}{ Isorhamnetin } & Apis mellifera & $\mathrm{BP}$ & Methanol; ethanol & UHPLC/MS-MS & Kostić et al. (2019) \\
\hline & $\begin{array}{l}\text { Stingless bee (Heterotrigona } \\
\text { itama) }\end{array}$ & BB & Methanol & LC-MS & Othman et al. (2020) \\
\hline \multirow[t]{2}{*}{ Isorhamnetin-3-O-glucoside } & Apis mellifera & $\mathrm{BP}$ & Methanol; ethanol & $\begin{array}{l}\text { UHPLC/MS-MS } \\
\text { Orbitrap }\end{array}$ & Kostić et al. (2019) \\
\hline & Apis mellifera iberiensis & $\mathrm{BB}$ & Methanol & HPLC-DAD-ESI/MS & Sobral et al. (2017) \\
\hline Isorhamnetin-di-3,7-O-glucoside & Apis mellifera & $\mathrm{BP}$ & $\begin{array}{l}\text { Dichloromethane; } \\
\text { methanol/water }\end{array}$ & $\begin{array}{l}\text { RP-HPLC-DAD-ESI- } \\
\text { MS/MS }\end{array}$ & Negri et al. (2018) \\
\hline $\begin{array}{l}\text { Isorhamnetin-3-O-(6"-O-p- } \\
\text { coumaroyl)-glucoside }\end{array}$ & Apis mellifera & $\mathrm{BP}$ & $\begin{array}{l}\text { Dichloromethane; } \\
\text { methanol/water }\end{array}$ & $\begin{array}{l}\text { RP-HPLC-DAD-ESI- } \\
\text { MS/MS }\end{array}$ & Negri et al. (2018) \\
\hline $\begin{array}{l}\text { Isorhamnetin-3-O-(2"-O- } \\
\text { rhamnosyl)-glucoside }\end{array}$ & Apis mellifera & $\mathrm{BP}$ & $\begin{array}{l}\text { Dichloromethane; } \\
\text { methanol/water }\end{array}$ & $\begin{array}{l}\text { RP-HPLC-DAD-ESI- } \\
\text { MS/MS }\end{array}$ & Negri et al. (2018) \\
\hline Acetyl isorhamnetin- $O$-hexoside & Apis mellifera iberiensis & $\mathrm{BB}$ & Methanol & HPLC-DAD-ESI/MS & Sobral et al. (2017) \\
\hline $\begin{array}{l}\text { Isorhamnetin-3-O-(2" }, 3^{\prime \prime}-O- \\
\text { dirhamnosyl)-glucoside }\end{array}$ & Apis mellifera & $\mathrm{BP}$ & $\begin{array}{l}\text { Dichloromethane; } \\
\text { methanol/water }\end{array}$ & $\begin{array}{l}\text { RP-HPLC-DAD-ESI- } \\
\text { MS/MS }\end{array}$ & Negri et al. (2018) \\
\hline $\begin{array}{l}\text { Isorhamnetin-3-O-(2" }-O \text {-rhamnosyl } \\
\text { acetyl) glucoside }\end{array}$ & Apis mellifera & $\mathrm{BP}$ & $\begin{array}{l}\text { Dichloromethane; } \\
\text { methanol/water }\end{array}$ & $\begin{array}{l}\text { RP-HPLC-DAD-ESI- } \\
\text { MS/MS }\end{array}$ & Negri et al. (2018) \\
\hline \multirow{2}{*}{$\begin{array}{l}\text { Isorhamnetin-3-O-rutinoside } \\
\text { (Narcissin) }\end{array}$} & Apis mellifera & $\mathrm{BP}$ & Methanol; ethanol & UHPLC/MS-MS & Kostić et al. (2019) \\
\hline & n.i. & $\mathrm{BB}$ & Methanol & LC-DAD-ESI/MS & Bakour et al. (2019) \\
\hline Isorhamnetin-O-dihexoside & n.i. & $\mathrm{BP}$ & Ethanol & UHPLC-DAD-ESI-MS & Anjos, Fernandes, et al. (2019) \\
\hline $\begin{array}{l}\text { Isorhamnetin- } O \text {-hexosyl-O- } \\
\text { rutinoside }\end{array}$ & n.i. & $\mathrm{BB}$ & Methanol & LC-DAD-ESI/MS & Bakour et al. (2019) \\
\hline Isorhamnetin-3-O-rhamnoside & Apis mellifera iberiensis & BB & Methanol & HPLC-DAD-ESI/MS & Sobral et al. (2017) \\
\hline $\begin{array}{l}\text { Isorhamnetin- } O \text {-(malonyl)- } \\
\text { hexoside isomer } 1\end{array}$ & n.i. & $\mathrm{BP}$ & Ethanol & UHPLC-DAD-ESI-MS & Anjos, Fernandes, et al. (2019) \\
\hline $\begin{array}{l}\text { Isorhamnetin- } O \text {-(malonyl)- } \\
\text { hexoside isomer } 2\end{array}$ & n.i. & $\mathrm{BP}$ & Ethanol & UHPLC-DAD-ESI-MS & Anjos, Fernandes, et al. (2019) \\
\hline Isorhamnetin-O-pentosyl-hexoside & Apis mellifera iberiensis & BB & Methanol & $\begin{array}{l}\text { LC-DAD-ESI/MS; } \\
\text { HPLC-DAD-ESI/MS }\end{array}$ & Bakour et al. (2019); Sobral et al. (2017) \\
\hline $\begin{array}{l}\text { Isorhamnetin-O-rhamnoside- } \\
\text { hexoside }\end{array}$ & n.i. & $\mathrm{BB}$ & Methanol & LC-DAD-ESI/MS & Bakour et al. (2019) \\
\hline \multirow[t]{2}{*}{ Kaempferol } & Apis mellifera & $\mathrm{BP}$ & Methanol; ethanol & $\begin{array}{l}\text { UHPLC/MS-MS; } \\
\text { HPLC-PDA }\end{array}$ & $\begin{array}{l}\text { De-Melo, Estevinho, Moreira, } \\
\text { Delerue-Matos, Freitas, et al. (2018); Kostić } \\
\text { et al. (2019) }\end{array}$ \\
\hline & $\begin{array}{l}\text { Apis mellifera/Stingless bee } \\
\text { (Heterotrigona itama) }\end{array}$ & BB & Methanol & LC-MS & $\begin{array}{l}\text { Čeksteryte et al. (2016); Othman et al. } \\
\text { (2020) }\end{array}$ \\
\hline $\begin{array}{l}\text { Acetyl kaempferol-O- } \\
\text { deoxyhexosyl-hexoside }\end{array}$ & Apis mellifera iberiensis & BB & Methanol & HPLC-DAD-ESI/MS & Sobral et al. (2017) \\
\hline $\begin{array}{l}\text { Kaempferol-3-O-glucosyl- } \\
\text { rutinoside }\end{array}$ & Apis mellifera & BP & Ethanol & HPLC-MS & $\begin{array}{l}\text { De-Melo, Estevinho, Moreira, } \\
\text { Delerue-Matos, Freitas, et al. (2018) }\end{array}$ \\
\hline $\begin{array}{l}\text { Kaempferol-O-pentosyl- } \\
\text { deoxyhexoside }\end{array}$ & Apis mellifera iberiensis & BB & Methanol & HPLC-DAD-ESI/MS & Sobral et al. (2017) \\
\hline $\begin{array}{l}\text { Kaempferol-3-O-rhamnosyl- } \\
\text { glucoside }\end{array}$ & Apis mellifera & $\mathrm{BP}$ & Ethanol & HPLC-MS & $\begin{array}{l}\text { De-Melo, Estevinho, Moreira, } \\
\text { Delerue-Matos, Freitas, et al. (2018) }\end{array}$ \\
\hline
\end{tabular}

(continued on next page) 
Table 3 (continued)

\begin{tabular}{|c|c|c|c|c|c|}
\hline Phenolic compounds & Bee specie & Source & Extraction solvent & Spectral analysis & References \\
\hline Kaempferol-3-O-rhamnoside & Apis mellifera iberiensis & BB & Methanol & HPLC-DAD-ESI/MS & Sobral et al. (2017) \\
\hline Kaempferol-7-O-rutinoside & Apis mellifera & $\mathrm{BP}$ & Ethanol & HPLC-MS & $\begin{array}{l}\text { De-Melo, Estevinho, Moreira, } \\
\text { Delerue-Matos, Freitas, et al. (2018) }\end{array}$ \\
\hline $\begin{array}{l}\text { Kaempferol-O-hexosyl-O- } \\
\text { rutinoside }\end{array}$ & n.i. & BB & Methanol & LC-DAD-ESI/MS & Bakour et al. (2019) \\
\hline Kaempferol-3-O-rutinoside & n.i. & BB & Methanol & LC-DAD-ESI/MS & Bakour et al. (2019) \\
\hline Kaempferol-O-dihexoside & Apis mellifera iberiensis & BB & Methanol & HPLC-DAD-ESI/MS & Sobral et al. (2017) \\
\hline Kaempferol-3-O-glycoside & $\begin{array}{l}\text { Apis mellifera and Apis } \\
\text { dorsata }\end{array}$ & BB & Ethanol & HPLC/DAD & Urcan et al. (2018) \\
\hline Galangin & Apis mellifera & $\mathrm{BP}$ & Methanol; ethanol & UHPLC/MS-MS & Kostić et al. (2019) \\
\hline \multirow[t]{2}{*}{ Myricetin } & n.i. & $\mathrm{BP}$ & Ethanol & UHPLC-DAD-ESI-MS & Anjos, Fernandes, et al. (2019) \\
\hline & $\begin{array}{l}\text { Apis mellifera and Apis } \\
\text { dorsata }\end{array}$ & BB & Ethanol & HPLC/DAD & Urcan et al. (2018) \\
\hline Myricetin-O-rutinoside & n.i. & BP & Ethanol & UHPLC-DAD-ESI-MS & Anjos, Fernandes, et al. (2019) \\
\hline Myricetin-3-O-rutinoside & Apis mellifera iberiensis & BB & Methanol & HPLC-DAD-ESI/MS & Sobral et al. (2017) \\
\hline Myricetin-3-O-glucoside & Apis mellifera iberiensis & BB & Methanol & HPLC-DAD-ESI/MS & Sobral et al. (2017) \\
\hline Myricetin- $O$-hexoside & n.i. & $\mathrm{BP}$ & Ethanol & UHPLC-DAD-ESI-MS & Anjos, Fernandes, et al. (2019) \\
\hline Myricetin-O-(malonyl)-rutinoside & n.i. & $\mathrm{BP}$ & Ethanol & UHPLC-DAD-ESI-MS & Anjos, Fernandes, et al. (2019) \\
\hline Myricetin-O-(malonyl)-hexoside & n.i. & $\mathrm{BP}$ & Ethanol & UHPLC-DAD-ESI-MS & Anjos, Fernandes, et al. (2019) \\
\hline $\begin{array}{l}\text { Myricetin-3- } O-\alpha-\mathrm{L}- \\
\text { rhamnopyranoside }\end{array}$ & Apis mellifera & $\mathrm{BP}$ & Ethanol & HPLC-MS & $\begin{array}{l}\text { De-Melo, Estevinho, Moreira, } \\
\text { Delerue-Matos, Freitas, et al. (2018) }\end{array}$ \\
\hline Patuletin-3-O-rhamnosylglucoside & Apis mellifera & $\mathrm{BP}$ & Ethanol & HPLC-MS & $\begin{array}{l}\text { De-Melo, Estevinho, Moreira, } \\
\text { Delerue-Matos, Freitas, et al. (2018) }\end{array}$ \\
\hline Methyl herbacetrin-O-dihexoside & n.i. & BB & Methanol & LC-DAD-ESI/MS & Bakour et al. (2019) \\
\hline Methyl herbacetrin-3-O-rutinoside & n.i. & BB & Methanol & LC-DAD-ESI/MS & Bakour et al. (2019) \\
\hline Herbacetin-3-O-glycoside & $\begin{array}{l}\text { Apis mellifera and Apis } \\
\text { dorsata }\end{array}$ & BB & Ethanol & HPLC/DAD & Urcan et al. (2018) \\
\hline $\begin{array}{l}\text { Methyl herbacetrin-O-hexosyl- } \\
\text { rutinoside }\end{array}$ & Apis mellifera iberiensis & BB & Methanol & HPLC-DAD-ESI/MS & Sobral et al. (2017) \\
\hline $\begin{array}{l}\text { Methyl herbacetrin-3-O-glucoside } \\
\text { Flavanols }\end{array}$ & Apis mellifera iberiensis & BB & Methanol & HPLC-DAD-ESI/MS & Sobral et al. (2017) \\
\hline Procyanidin dimer B1 & Apis mellifera & $\mathrm{BP}$ & Ethanol & HPLC-MS & $\begin{array}{l}\text { De-Melo, Estevinho, Moreira, } \\
\text { Delerue-Matos, Freitas, et al. (2018) }\end{array}$ \\
\hline Catechin & Apis mellifera & $\mathrm{BP}$ & Ethanol; methanol & HPLC-PDA & $\begin{array}{l}\text { De-Melo, Estevinho, Moreira, } \\
\text { Delerue-Matos, Freitas, et al. (2018) }\end{array}$ \\
\hline Epicatechin & Apis mellifera & BP & Ethanol; methanol & HPLC-PDA & $\begin{array}{l}\text { De-Melo, Estevinho, Moreira, } \\
\text { Delerue-Matos, Freitas, et al. (2018) }\end{array}$ \\
\hline \multicolumn{6}{|l|}{ Flavanonols } \\
\hline Pinobanksin-3-O-butyrate & Apis mellifera & BP & Ethanol & HPLC-MS & $\begin{array}{l}\text { De-Melo, Estevinho, Moreira, } \\
\text { Delerue-Matos, Freitas, et al. (2018) }\end{array}$ \\
\hline Pinobanksin-3-O-propionate & Apis mellifera & $\mathrm{BP}$ & Ethanol & HPLC-MS & $\begin{array}{l}\text { De-Melo, Estevinho, Moreira, } \\
\text { Delerue-Matos, Freitas, et al. (2018) }\end{array}$ \\
\hline Taxifolin & Apis mellifera & BP & Methanol; ethanol & UHPLC/MS-MS & Kostić et al. (2019) \\
\hline \multicolumn{6}{|l|}{ Dihydroflavonols } \\
\hline Dihydromyricetin & Apis mellifera & BP & Ethanol & HPLC-MS & $\begin{array}{l}\text { De-Melo, Estevinho, Moreira, } \\
\text { Delerue-Matos, Freitas, et al. (2018) }\end{array}$ \\
\hline $\begin{array}{l}\text { Pinobanksin-5-methylether-3-O- } \\
\text { acetate }\end{array}$ & Apis mellifera & $\mathrm{BP}$ & Ethanol & HPLC-MS & $\begin{array}{l}\text { De-Melo, Estevinho, Moreira, } \\
\text { Delerue-Matos, Freitas, et al. (2018) }\end{array}$ \\
\hline Dihydroquercetin-3-O-rhamnoside & Apis mellifera & BP & Ethanol & HPLC-MS & $\begin{array}{l}\text { De-Melo, Estevinho, Moreira, } \\
\text { Delerue-Matos, Freitas, et al. (2018) }\end{array}$ \\
\hline Dihydroquercetin & n.i. & BP & Methanol & UHPLC-ESI-QTOF & $\begin{array}{l}\text { Rocchetti, Castiglioni, Maldarizzi, Carloni, } \\
\text { and Lucini (2019) }\end{array}$ \\
\hline \multicolumn{6}{|l|}{ Flavanones } \\
\hline \multirow[t]{2}{*}{ Naringin } & Apis mellifera & BP & Ethanol; methanol & HPLC-PDA & $\begin{array}{l}\text { De-Melo, Estevinho, Moreira, } \\
\text { Delerue-Matos, Freitas, et al. (2018) }\end{array}$ \\
\hline & n.i. & BB & Ethanol & HPLC & $\begin{array}{l}\text { Tavdidishvili, Khutsidze, Pkhakadze, } \\
\text { Vanidze, and Kalandia (2014) }\end{array}$ \\
\hline Naringenin & Apis mellifera & BP & Methanol; ethanol & $\begin{array}{l}\text { UHPLC/MS-MS; } \\
\text { SPME-GC/MS }\end{array}$ & Kostić et al. (2019); LeBlanc et al. (2009) \\
\hline Naringin $6^{\prime}$-malonate & n.i. & BP & Methanol & UHPLC-ESI-QTOF & Rocchetti et al. (2019) \\
\hline Naringenin hexoside & Apis mellifera & BP & Ethanol & HPLC-MS & $\begin{array}{l}\text { De-Melo, Estevinho, Moreira, } \\
\text { Delerue-Matos, Freitas, et al. (2018) }\end{array}$ \\
\hline $\begin{array}{l}4^{\prime}, 5 \text {-dihydroxy-7- } \\
\text { methoxyflavanone }\end{array}$ & n.i. & BP & Methanol & SPME-GC/MS & LeBlanc et al. (2009) \\
\hline Eriodictyol & Apis mellifera & BP & Ethanol & HPLC-MS & $\begin{array}{l}\text { De-Melo, Estevinho, Moreira, } \\
\text { Delerue-Matos, Freitas, et al. (2018) }\end{array}$ \\
\hline Naringin-4'-O-glucoside & n.i. & $\mathrm{BP}$ & Methanol & UHPLC-ESI-QTOF & Rocchetti et al. (2019) \\
\hline Naringenin-7-O-glucoside & n.i. & $\mathrm{BP}$ & Methanol & UHPLC-ESI-QTOF & Rocchetti et al. (2019) \\
\hline Hesperidin & n.i. & BP & Ethyl acetate & HPLC & Fanali, Dugo, and Rocco (2013) \\
\hline \multicolumn{6}{|l|}{ Dihydrochalcones } \\
\hline Phloretin & Apis mellifera & BP & Methanol; ethanol & UHPLC/MS-MS & Kostić et al. (2019) \\
\hline \multicolumn{6}{|l|}{ Flavones } \\
\hline \multirow[t]{3}{*}{ Luteolin } & Apis mellifera & BP & Methanol; ethanol & UHPLC/MS-MS & Kostić et al. (2019) \\
\hline & $\begin{array}{l}\text { Apis mellifera and Apis } \\
\text { dorsata }\end{array}$ & $\mathrm{BB}$ & Ethanol & HPLC/DAD & Urcan et al. (2018) \\
\hline & n.i. & BP & Methanol & UHPLC-ESI-QTOF & Rocchetti et al. (2019) \\
\hline
\end{tabular}


Table 3 (continued)

\begin{tabular}{|c|c|c|c|c|c|}
\hline Phenolic compounds & Bee specie & Source & Extraction solvent & Spectral analysis & References \\
\hline \multicolumn{6}{|l|}{$\begin{array}{l}\text { Apigenin 7-O-(6"-malonyl-apiosyl- } \\
\text { glucoside) }\end{array}$} \\
\hline \multirow[t]{2}{*}{ Apigenin } & Apis mellifera & $\mathrm{BP}$ & Methanol; ethanol & UHPLC/MS-MS & Kostić et al. (2019) \\
\hline & $\begin{array}{l}\text { Apis mellifera/Stingless bee } \\
\text { (Heterotrigona itama) }\end{array}$ & BB & Methanol & $\begin{array}{l}\text { LC-MS; HPLC/UV/ } \\
\text { MS }\end{array}$ & $\begin{array}{l}\text { Baltrušaityte et al. (2007); Othman et al. } \\
\text { (2020) }\end{array}$ \\
\hline Apigenin 6-C-glucoside & n.i. & BP & Methanol & UHPLC-ESI-QTOF & Rocchetti et al. (2019) \\
\hline Tricetin & $\begin{array}{l}\text { Apis mellifera and Apis } \\
\text { dorsata }\end{array}$ & $\mathrm{BB}$ & Ethanol & HPLC/DAD & Urcan et al. (2018) \\
\hline Tricetin-7-O-(pentoside-glucoside) & Apis mellifera & $\mathrm{BP}$ & Ethanol & HPLC-MS & $\begin{array}{l}\text { De-Melo, Estevinho, Moreira, } \\
\text { Delerue-Matos, Freitas, et al. (2018) }\end{array}$ \\
\hline Acacetin & Apis mellifera & $\mathrm{BP}$ & Methanol; ethanol & UHPLC/MS-MS & Kostić et al. (2019) \\
\hline Rutin & n.i. & BB & Ethanol & HPLC & Tavdidishvili et al. (2014) \\
\hline \multirow[t]{2}{*}{ Chrysin } & n.i. & $\mathrm{BP}$ & Methanol & HPLC-PDA & Ketkar et al. (2014) \\
\hline & n.i. & BB & Methanol & HPLC/UV/MS & Baltrušaitytè et al. (2007) \\
\hline Genkwanin & Apis mellifera & $\mathrm{BP}$ & Methanol; ethanol & UHPLC/MS-MS & Kostić et al. (2019) \\
\hline Luteolin-O-dihexoside & n.i. & BP & Ethanol & UHPLC-DAD-ESI-MS & Anjos, Fernandes, et al. (2019) \\
\hline Luteolin 7-O-glucuronide & n.i. & $\mathrm{BP}$ & Methanol & UHPLC-ESI-QTOF & Rocchetti et al. (2019) \\
\hline Luteolin-di-O-hexosyl-rhamnoside & n.i. & BP & Ethanol & UHPLC-DAD-ESI-MS & Anjos, Fernandes, et al. (2019) \\
\hline Luteolin-O-(malonyl)-hexoside & n.i. & $\mathrm{BP}$ & Ethanol & UHPLC-DAD-ESI-MS & Anjos, Fernandes, et al. (2019) \\
\hline Luteolin-7-O-6" -acetylglucoside & Apis mellifera & $\mathrm{BP}$ & Ethanol & HPLC-MS & $\begin{array}{l}\text { De-Melo, Estevinho, Moreira, } \\
\text { Delerue-Matos, Freitas, et al. (2018) }\end{array}$ \\
\hline Orientin-2-O-xyloside & Apis mellifera & $\mathrm{BP}$ & Ethanol & HPLC-MS & $\begin{array}{l}\text { De-Melo, Estevinho, Moreira, } \\
\text { Delerue-Matos, Freitas, et al. (2018) }\end{array}$ \\
\hline Tetramethylscutellarein & n.i. & BP & Methanol & UHPLC-ESI-QTOF & Rocchetti et al. (2019) \\
\hline Laricitrin-3-O-rhamnoside & Apis mellifera iberiensis & BB & Methanol & HPLC-DAD-ESI/MS & Sobral et al. (2017) \\
\hline \multicolumn{6}{|l|}{ Anthocyanins } \\
\hline Cyanidin-3-rutinoside & Apis mellifera & $\mathrm{BP}$ & Ethanol & HPLC-MS & $\begin{array}{l}\text { De-Melo, Estevinho, Moreira, } \\
\text { Delerue-Matos, Freitas, et al. (2018) }\end{array}$ \\
\hline 6-O-caffeoyl glucoside & Apis mellifera & $\mathrm{BP}$ & $\begin{array}{l}\text { Dichloromethane; } \\
\text { methanol/water }\end{array}$ & $\begin{array}{l}\text { RP-HPLC-DAD-ESI- } \\
\text { MS/MS }\end{array}$ & Negri et al. (2018) \\
\hline Petunidin-3-O-galactoside & Apis mellifera & BP & Ethanol & HPLC-MS & $\begin{array}{l}\text { De-Melo, Estevinho, Moreira, } \\
\text { Delerue-Matos, Freitas, et al. (2018) }\end{array}$ \\
\hline Petunidin 3-O-arabinoside & n.i. & $\mathrm{BP}$ & Methanol & UHPLC-ESI-QTOF & Rocchetti et al. (2019) \\
\hline Cyanidin 3-O-xyloside/arabinoside & n.i. & $\mathrm{BP}$ & Methanol & UHPLC-ESI-QTOF & Rocchetti et al. (2019) \\
\hline $\begin{array}{l}\text { Delphinidin 3-O-(6"-p-coumaroyl- } \\
\text { glucoside })\end{array}$ & n.i. & $\mathrm{BP}$ & Methanol & UHPLC-ESI-QTOF & Rocchetti et al. (2019) \\
\hline Pelargonidin 3-O-glucoside & n.i. & BP & Methanol & UHPLC-ESI-QTOF & Rocchetti et al. (2019) \\
\hline Delphinidin 3-O-glucoside & n.i. & $\mathrm{BP}$ & Methanol & UHPLC-ESI-QTOF & Rocchetti et al. (2019) \\
\hline Delphinidin 3-O-glucosyl-glucoside & n.i. & BP & Methanol & UHPLC-ESI-QTOF & Rocchetti et al. (2019) \\
\hline Delphinidin 3-O-rutinoside & n.i. & $\mathrm{BP}$ & Methanol & UHPLC-ESI-QTOF & Rocchetti et al. (2019) \\
\hline Cyanidin 3-O-sophoroside & n.i. & $\mathrm{BP}$ & Methanol & UHPLC-ESI-QTOF & Rocchetti et al. (2019) \\
\hline \multicolumn{6}{|l|}{ Isoflavonoids } \\
\hline $7,8,2^{\prime}, 4^{\prime}$-Tetrahydroxyisoflavone & n.i. & $\mathrm{BP}$ & Methanol & SPME-GC/MS & LeBlanc et al. (2009) \\
\hline Formononetin & n.i. & BP & Methanol & UHPLC-ESI-QTOF & Rocchetti et al. (2019) \\
\hline Genistin & n.i. & $\mathrm{BP}$ & Methanol & UHPLC-ESI-QTOF & Rocchetti et al. (2019) \\
\hline \multicolumn{6}{|l|}{ Hydroxycinnamic acid } \\
\hline \multirow[t]{2}{*}{ Caffeic acid } & Apis mellifera & $\mathrm{BP}$ & Methanol; ethanol & UHPLC/MS-MS & Kostić et al. (2019) \\
\hline & & BB & Methanol & LC-MS & Othman et al. (2020) \\
\hline Caffeic acid 4-O-glucoside & n.i. & BP & Methanol & UHPLC-ESI-QTOF & Rocchetti et al. (2019) \\
\hline Hydrocaffeic acid & n.i. & $\mathrm{BP}$ & Ethanol & HPLC/ESI-MS & Karabagias et al. (2018) \\
\hline \multirow[t]{2}{*}{ p-coumaric acid } & Apis mellifera & $\mathrm{BP}$ & Methanol; ethanol & $\begin{array}{l}\text { UHPLC/MS-MS; } \\
\text { HPLC-PDA }\end{array}$ & $\begin{array}{l}\text { De-Melo, Estevinho, Moreira, } \\
\text { Delerue-Matos, Freitas, et al. (2018); Kostić } \\
\text { et al. (2019) }\end{array}$ \\
\hline & n.i. & BP & Methanol & HPLC/UV/MS & Baltrušaityte et al. (2007) \\
\hline Trihydroxycinnamic acid & Apis mellifera & $\mathrm{BP}$ & $\begin{array}{l}\text { Dichloromethane; } \\
\text { methanol/water }\end{array}$ & $\begin{array}{l}\text { RP-HPLC-DAD-ESI- } \\
\text { MS/MS }\end{array}$ & Negri et al. (2018) \\
\hline \multirow[t]{2}{*}{ Ferulic acid } & Apis mellifera & $\mathrm{BP}$ & Methanol; ethanol & UHPLC/MS-MS & Kostić et al. (2019) \\
\hline & $\begin{array}{l}\text { Stingless bee (Heterotrigona } \\
\text { itama) }\end{array}$ & BB & Methanol & LC-MS & Othman et al. (2020) \\
\hline Feruloyl glucose & n.i. & BP & Methanol & UHPLC-ESI-QTOF & Rocchetti et al. (2019) \\
\hline Sinapic acid & Apis mellifera & BP & Ethanol; methanol & HPLC-PDA & $\begin{array}{l}\text { De-Melo, Estevinho, Moreira, } \\
\text { Delerue-Matos, Freitas, et al. (2018) }\end{array}$ \\
\hline Cinnamic acid & Apis mellifera & BP & Ethanol; methanol & HPLC-PDA & $\begin{array}{l}\text { De-Melo, Estevinho, Moreira, } \\
\text { Delerue-Matos, Freitas, et al. (2018) }\end{array}$ \\
\hline 2-feruloyl-1-sinapoylgentiobiose & Apis mellifera & BP & Ethanol & HPLC-MS & $\begin{array}{l}\text { De-Melo, Estevinho, Moreira, } \\
\text { Delerue-Matos, Freitas, et al. (2018) }\end{array}$ \\
\hline Caffeoyl glucose & n.i. & $\mathrm{BP}$ & Methanol & UHPLC-ESI-QTOF & Rocchetti et al. (2019) \\
\hline$p$-coumaroyl tyrosine & n.i. & $\mathrm{BP}$ & Ethanol & HPLC/ESI-MS & Karabagias et al. (2018) \\
\hline $\begin{array}{l}\text { Hydroxycinnamic acid derivatives } \\
\quad 1,2,3,4,5\end{array}$ & $\begin{array}{l}\text { Apis mellifera and Apis } \\
\text { dorsata }\end{array}$ & BB & Ethanol & HPLC/DAD & Urcan et al. (2018) \\
\hline \multicolumn{6}{|l|}{ Hydroxybenzoic acids } \\
\hline Gallic acid & Apis mellifera & BP & Ethanol; methanol & HPLC-PDA & $\begin{array}{l}\text { De-Melo, Estevinho, Moreira, } \\
\text { Delerue-Matos, Freitas, et al. (2018) }\end{array}$ \\
\hline Vanillic acid & Apis mellifera & BP & Ethanol; methanol & HPLC-PDA & $\begin{array}{l}\text { De-Melo, Estevinho, Moreira, } \\
\text { Delerue-Matos, Freitas, et al. (2018) }\end{array}$ \\
\hline Syringic acid & Apis mellifera & BP & Ethanol; methanol & HPLC-PDA & \\
\hline
\end{tabular}


Table 3 (continued)

\begin{tabular}{|c|c|c|c|c|c|}
\hline Phenolic compounds & Bee specie & Source & Extraction solvent & Spectral analysis & References \\
\hline Ellagic acid & Apis mellifera & $\mathrm{BP}$ & Ethanol & HPLC-MS & $\begin{array}{l}\text { De-Melo, Estevinho, Moreira, } \\
\text { Delerue-Matos, Freitas, et al. (2018) } \\
\text { De-Melo, Estevinho, Moreira, } \\
\text { Delerue-Matos, Freitas, et al. (2018) }\end{array}$ \\
\hline \multicolumn{6}{|l|}{ Dihydroxybenzoic acids } \\
\hline Protocatechuic acid & Apis mellifera & BP & Ethanol; methanol & HPLC-PDA & $\begin{array}{l}\text { De-Melo, Estevinho, Moreira, } \\
\text { Delerue-Matos, Freitas, et al. (2018) }\end{array}$ \\
\hline$\beta$-resorcylic acid & Apis mellifera & $\mathrm{BP}$ & Ethanol; methanol & HPLC-PDA & $\begin{array}{l}\text { De-Melo, Estevinho, Moreira, } \\
\text { Delerue-Matos, Freitas, et al. (2018) }\end{array}$ \\
\hline \multicolumn{6}{|l|}{ Benzoic acids and derivatives } \\
\hline 4-methoxy benzoic & Apis mellifera & $\mathrm{BP}$ & Ethanol & GC-MS & Carpes et al. (2013) \\
\hline \multicolumn{6}{|l|}{ Other polyphenols } \\
\hline Aesculin & Apis mellifera & $\mathrm{BP}$ & Methanol; ethanol & UHPLC/MS-MS & Kostić et al. (2019) \\
\hline Urolithin B & n.i. & $\mathrm{BP}$ & Ethanol & HPLC/ESI-MS & Karabagias et al. (2018) \\
\hline Chlorogenic acid & Apis mellifera & $\mathrm{BP}$ & Ethanol; methanol & HPLC-PDA & $\begin{array}{l}\text { De-Melo, Estevinho, Moreira, } \\
\text { Delerue-Matos, Freitas, et al. (2018) }\end{array}$ \\
\hline Coumaroyl quinic acid & n.i. & $\mathrm{BP}$ & Ethanol & UHPLC-DAD-ESI-MS & Anjos, Fernandes, et al. (2019) \\
\hline Glucoraphanin & Apis mellifera & $\mathrm{BP}$ & Ethanol & HPLC-MS & $\begin{array}{l}\text { De-Melo, Estevinho, Moreira, } \\
\text { Delerue-Matos, Freitas, et al. (2018) }\end{array}$ \\
\hline 5-O-caffeoylquinic acid & Apis mellifera & $\mathrm{BP}$ & Methanol; ethanol & UHPLC/MS-MS & Kostić et al. (2019) \\
\hline Isopimpinellin & n.i. & $\mathrm{BP}$ & Ethanol & HPLC/ESI-MS & Karabagias et al. (2018) \\
\hline \multicolumn{6}{|l|}{ Polyamines } \\
\hline $\mathrm{N}^{\prime}, \mathrm{N}^{\prime \prime}, \mathrm{N}^{\prime \prime \prime}$-tris-caffeoyl spermidine & n.i. & $\mathrm{BP}$ & Ethanol/water & LC/DAD/ESI-MS & $\begin{array}{l}\text { El Ghouizi, El Menyiy, Falcão, Vilas-Boas, } \\
\text { and Lyoussi (2020) }\end{array}$ \\
\hline $\begin{array}{l}\mathrm{N}^{\prime}, \mathrm{N}^{\prime \prime} \text {-dicaffeoyl, } \mathrm{N}^{\prime \prime \prime} \text {-coumaroyl } \\
\text { spermidine }\end{array}$ & Apis mellifera & BP & $\begin{array}{l}\text { Dichloromethane; } \\
\text { methanol/water }\end{array}$ & $\begin{array}{l}\text { RP-HPLC-DAD-ESI- } \\
\text { MS/MS }\end{array}$ & Negri et al. (2018) \\
\hline $\begin{array}{l}\mathrm{N}^{\prime}, \mathrm{N}^{\prime \prime} \text {-dicaffeoyl, } \mathrm{N}^{\prime \prime \prime} \text {-feruloyl } \\
\text { spermidine }\end{array}$ & Apis mellifera & $\mathrm{BP}$ & $\begin{array}{l}\text { Dichloromethane; } \\
\text { methanol/water }\end{array}$ & $\begin{array}{l}\text { RP-HPLC-DAD-ESI- } \\
\text { MS/MS }\end{array}$ & Negri et al. (2018) \\
\hline $\begin{array}{l}\mathrm{N}^{\prime} \text {-caffeoyl-N" }{ }^{\prime \prime} \text {-feruloyl, } \mathrm{N}^{\prime \prime \prime} \text { - } \\
\text { coumaroyl spermidine }\end{array}$ & Apis mellifera & $\mathrm{BP}$ & $\begin{array}{l}\text { Dichloromethane; } \\
\text { methanol/water }\end{array}$ & $\begin{array}{l}\text { RP-HPLC-DAD-ESI- } \\
\text { MS/MS }\end{array}$ & Negri et al. (2018) \\
\hline $\begin{array}{l}\mathrm{N}^{\prime} \text {-caffeoyl- } \mathrm{N}^{\prime \prime}, \mathrm{N}^{\prime \prime \prime} \text { - dicoumaroyl } \\
\text { spermidine }\end{array}$ & n.i. & $\mathrm{BP}$ & Ethanol/water & LC/DAD/ESI-MS & El Ghouizi et al. (2020) \\
\hline $\begin{array}{l}\mathrm{N}^{\prime}, \mathrm{N}^{\prime \prime}, \mathrm{N}^{\prime \prime \prime} \text {-tris-p-coumaroyl } \\
\text { spermidine }\end{array}$ & Apis mellifera & $\mathrm{BP}$ & $\begin{array}{l}\text { Dichloromethane; } \\
\text { methanol/water }\end{array}$ & $\begin{array}{l}\text { RP-HPLC-DAD-ESI- } \\
\text { MS/MS }\end{array}$ & Negri et al. (2018) \\
\hline $\mathrm{N}^{\prime}, \mathrm{N}^{\prime \prime}, \mathrm{N}^{\prime \prime \prime}$-tris-p-feruloyl spermidine & Apis mellifera & $\mathrm{BP}$ & $\begin{array}{l}\text { Dichloromethane; } \\
\text { methanol/water }\end{array}$ & $\begin{array}{l}\text { RP-HPLC-DAD-ESI- } \\
\text { MS/MS }\end{array}$ & Negri et al. (2018) \\
\hline \multicolumn{6}{|l|}{ Tyrosols } \\
\hline \multicolumn{6}{|l|}{ Curcuminoids } \\
\hline Curcumin & n.i. & $\mathrm{BP}$ & Methanol & UHPLC-ESI-QTOF & Rocchetti et al. (2019) \\
\hline \multicolumn{6}{|l|}{ Lignans } \\
\hline Sesamol & n.i. & $\mathrm{BP}$ & Methanol & UHPLC-ESI-QTOF & Rocchetti et al. (2019) \\
\hline
\end{tabular}

n.i., no information.

cholesterol, collagen and some hormones (Grosso et al., 2013). Hence it should be taken regularly with the diet. According to the dietary reference intake, daily vitamin $C$ intake is $90 \mathrm{mg}$ for men and $75 \mathrm{mg}$ for women (Russell et al., 2001). Vitamin C has many different therapeutic properties, such as anticarcinogenic, improving neurotransmission and slowing macular degeneration (Evans \& Lawrenson, 2017). Also, there are several research's investigating whether vitamin $\mathrm{C}$ has any effect on cardiovascular diseases. Sesso et al. (2008) investigated if long-term vitamin C intake reduced the risk of major cardiovascular diseases, revealing that there is no effect in the short or long term vitamin $\mathrm{C}$ intake.

\section{Digestion and bioavailability of phytochemicals in bee pollen and bee bread}

The concept of phytochemicals bioavailability comprises its release from the food matrix, their alteration in the GIT, bioaccessibility, absorption, tissue distribution with systemic circulation, and finally their effect (i.e. bioactivity) on the tissues (Stahl et al., 2002). The bioaccessibility depends largely in the effectiveness of the digestion which may occur at three different levels, in the mouth, stomach or in the intestines. In the oral stage, when chewed, the food is mixed with saliva containing $\alpha$-amylase and lingual lipase enzymes initiating several degradation processes such as the hydrolyze of carbohydrates (C. Li, Yu, Wu, \& Chen, 2020). The gastric digestion starts with the decrease of $\mathrm{pH}$ due to the secretion of hydrochloric acid, followed by the action of enzymes such as pepsin and gastric lipase, and finally the mechanical digestion due to the contraction activity of the stomach (C. Li et al., 2020). The intestinal phase involves also the action of enzymes produced by the pancreas to digest proteins, fats and carbohydrates, but also bile salts and hormones that regulate several metabolisms of sugars and fats. This stage occurs along the small intestine, through mixing and transport of intraluminal contents and absorption of nutrients (Yonekura \& Nagao, 2007).

With today's advanced analytical methods and instrumentation, it is possible to measure the bioavailability of dietary phytochemicals, however, some practical limitations may occur in the access of the bioaccessibility and biological activities in living systems. The interaction of phytochemicals with other compounds like proteins and carbohydrates in the GIT can alter their bioavailability. Besides, these can be absorbed and metabolized directly by the intestinal cells in the colon without passing to plasma and urine (Carbonell-Capella et al., 2014; Crozier et al., 2009). In the case of BP and BB, the strong multi-layered structure of pollen grains limits the release of bioactive compounds from its interior and the interaction of digestive enzymes with these food components (Bogdanov, 2011). Thereby the morphology of dietary foods significantly affects the degree of digestion and, consequently, bioavailability, which, in the case BP and BB, may require the use of specific methods to increase digestibility, as referred in more detail in section 5 . 
Table 4

Carotenoids existing in bee pollen and bee bread, their amounts and botanical origins.

\begin{tabular}{|c|c|c|c|c|c|c|}
\hline Carotenoids & $\begin{array}{l}\text { Bee } \\
\text { specie }\end{array}$ & $\begin{array}{l}\text { Bee } \\
\text { product }\end{array}$ & Botanical source & $\begin{array}{l}\text { Range } \\
\text { quantification }\end{array}$ & Spectral analysis & References \\
\hline \multicolumn{7}{|l|}{ Carotenes } \\
\hline \multirow[t]{5}{*}{$\beta$-Carotene } & n.i. & $\mathrm{BP}$ & $\begin{array}{l}\text { Asteraceae, Fabaceae, Brassicaceae, Rubiaceae, } \\
\text { Caesalpineaceae, Caricaceae, Ulmaceae, Myrtaceae, } \\
\text { Aquifoliaceae, Anacardiaceae, Melastomataceae, } \\
\text { Mimosaceae, Loranthaceae, Piperaceae, Poaceae, Burseraceae } \\
\text { and Rosaceae }\end{array}$ & $0.5-112.7 \mu \mathrm{g} / \mathrm{g}$ & HPLC & Sattler et al. (2015) \\
\hline & n.i. & $\mathrm{BP}$ & $\begin{array}{l}\text { Cistus ladanifer, Echium, Achillea, Taraxacum, Carduus, Cirsium, } \\
\text { Vicia, Quercus ilex, Quercus r., Rubus, Pinaceae, Filipendula, } \\
\text { Trifolium incarnatum, Trifolium pratense, Trifolium repens, } \\
\text { Prunus, Pyrus, Malus and Oxalis }\end{array}$ & n.i. & UHPLC-DAD & Gardana et al. (2018) \\
\hline & $\begin{array}{l}\text { Apis } \\
\text { mellifera }\end{array}$ & $\mathrm{BP}$ & n.i. & $\begin{array}{l}3.14-77.88 \mu \mathrm{g} / \\
\mathrm{g}\end{array}$ & HPLC & $\begin{array}{l}\text { Melo and } \\
\text { Almeida-Muradian } \\
\text { (2010) }\end{array}$ \\
\hline & n.i. & $\mathrm{BP}$ & $\begin{array}{l}\text { Rosaceae, Fabaceae, Asteraceae, Brasicaceae, Ericaceae and } \\
\text { Salicaceae }\end{array}$ & $\begin{array}{l}0.17-18.18 \mu \mathrm{g} / \\
\mathrm{g}\end{array}$ & HPLC-PDA & Mărgă;oan et al. (2014) \\
\hline & n.i. & BB & Dandelion, horse-chestnut, pine, heather,fireweed and birch & n.i. & TLC & $\begin{array}{l}\text { Barene, Daberte, and } \\
\text { Siksna (2015) }\end{array}$ \\
\hline$\alpha$-Carotene & n.i. & $\mathrm{BP}$ & $\begin{array}{l}\text { Asteraceae, Myrtaceae, Fabaceae, Loranthaceae, } \\
\text { Aquifoliaceae, Arecaceae, Piperaceae, Anacardiaceae, } \\
\text { Rubiaceae and Burseraceae }\end{array}$ & $3.3-324.7 \mu \mathrm{g} / \mathrm{g}$ & HPLC & Sattler et al. (2015) \\
\hline$\gamma$-Carotene & $\begin{array}{l}\text { Apis } \\
\text { mellifera }\end{array}$ & $\mathrm{BP}$ & Sunflower, clover, sesame and maize & $\begin{array}{l}53.81-128.7 \\
\mathrm{mg} / \mathrm{g}\end{array}$ & Spectrophotometric & $\begin{array}{l}\text { Abd Alla and Salem } \\
(2020)\end{array}$ \\
\hline$\xi$ - Carotene & & & & $\begin{array}{l}44.95-115.81 \\
\mathrm{mg} / \mathrm{g}\end{array}$ & & $\begin{array}{l}\text { Abd Alla and Salem } \\
(2020)\end{array}$ \\
\hline$\varepsilon$ - Carotene & & & & $\begin{array}{l}58.06-123.91 \\
\mathrm{mg} / \mathrm{g}\end{array}$ & & $\begin{array}{l}\text { Abd Alla and Salem } \\
(2020)\end{array}$ \\
\hline Lycopene & & & & $\begin{array}{l}38.44-121.5 \\
\mathrm{mg} / \mathrm{g}\end{array}$ & & $\begin{array}{l}\text { Abd Alla and Salem } \\
(2020)\end{array}$ \\
\hline & $\begin{array}{l}\text { Apis } \\
\text { mellifera }\end{array}$ & $\mathrm{BP}$ & Multifloral and monofloral bee pollen & $\begin{array}{l}0.59-16.17 \mu \mathrm{g} / \\
\mathrm{g}\end{array}$ & UHPLC-DAD & Bohoyo-Gil et al. (2012) \\
\hline \multicolumn{7}{|l|}{ Xanthophylls } \\
\hline \multirow[t]{3}{*}{ Zeaxanthin } & n.i. & $\mathrm{BP}$ & $\begin{array}{l}\text { Cistus ladanifer, Echium, Achillea, Taraxacum, Carduus, Cirsium, } \\
\text { Vicia, Quercus ilex, Quercus r., Rubus, Pinaceae, Filipendula, } \\
\text { Trifolium incarnatum, Trifolium pratense, Trifolium repens, } \\
\text { Prunus, Pyrus, Malus and Oxalis }\end{array}$ & n.i. & UHPLC-DAD & Gardana et al. (2018) \\
\hline & $\begin{array}{l}\text { Apis } \\
\text { mellifera }\end{array}$ & BP & Multifloral and monofloral bee pollen & $0.14-2.26 \mu \mathrm{g} / \mathrm{g}$ & UHPLC-DAD & Bohoyo-Gil et al. (2012) \\
\hline & $\begin{array}{l}\text { Apis } \\
\text { mellifera }\end{array}$ & $\mathrm{BP}$ & Sunflower, clover, sesame and maize & $\begin{array}{l}48.79-312.43 \\
\mathrm{mg} / \mathrm{g}\end{array}$ & Spectrophotometric & $\begin{array}{l}\text { Abd Alla and Salem } \\
(2020)\end{array}$ \\
\hline \multirow[t]{3}{*}{ Lutein } & n.i. & $\mathrm{BP}$ & $\begin{array}{l}\text { Rosaceae, Fabaceae, Asteraceae, Brasicaceae, Ericaceae and } \\
\text { Salicaceae }\end{array}$ & $\begin{array}{l}44.52-476.30 \\
\mu \mathrm{g} / \mathrm{g}\end{array}$ & HPLC-PDA & Mărgă;oan et al. (2014) \\
\hline & $\begin{array}{l}\text { Apis } \\
\text { mellifera }\end{array}$ & $\mathrm{BP}$ & Multifloral and monofloral bee pollen & $0.14-0.31 \mu \mathrm{g} / \mathrm{g}$ & UHPLC-DAD & Bohoyo-Gil et al. (2012) \\
\hline & n.i. & $\mathrm{BP}$ & $\begin{array}{l}\text { Cistus ladanifer, Echium, Achillea, Taraxacum, Carduus, Cirsium, } \\
\text { Vicia, Quercus ilex, Quercus r., Rubus, Pinaceae, Filipendula, } \\
\text { Trifolium incarnatum, Trifolium pratense, Trifolium repens, } \\
\text { Prunus, Pyrus, Malus and Oxalis }\end{array}$ & n.i. & UHPLC-DAD & Gardana et al. (2018) \\
\hline Capsantine & $\begin{array}{l}\text { Apis } \\
\text { mellifera }\end{array}$ & BP & Multifloral and monofloral bee pollen & $0.20-2.04 \mu \mathrm{g} / \mathrm{g}$ & UHPLC-DAD & Bohoyo-Gil et al. (2012) \\
\hline \multirow[t]{3}{*}{$\beta$-cryptoxanthin } & $\begin{array}{l}\text { Apis } \\
\text { mellifera }\end{array}$ & $\mathrm{BP}$ & Sunflower, clover, sesame and maize & $\begin{array}{l}40.77-85.42 \\
\mathrm{mg} / \mathrm{g}\end{array}$ & Spectrophotometric & $\begin{array}{l}\text { Abd Alla and Salem } \\
(2020)\end{array}$ \\
\hline & n.i. & BP & $\begin{array}{l}\text { Rosaceae, Fabaceae, Asteraceae, Brasicaceae, Ericaceae and } \\
\text { Salicaceae }\end{array}$ & $\begin{array}{l}1.02-35.43 \mu \mathrm{g} / \\
\mathrm{g}\end{array}$ & HPLC-PDA & Mărgă;oan et al. (2014) \\
\hline & $\begin{array}{l}\text { Apis } \\
\text { mellifera }\end{array}$ & BP & Multifloral and monofloral bee pollen & $\begin{array}{l}0.59-16.17 \mu g / \\
g\end{array}$ & UHPLC-DAD & Bohoyo-Gil et al. (2012) \\
\hline Isocryptoxanthin & $\begin{array}{l}\text { Apis } \\
\text { mellifera }\end{array}$ & $\mathrm{BP}$ & Sunflower, clover, sesame and maize & $\begin{array}{l}31.18-80.59 \\
\mathrm{mg} / \mathrm{g}\end{array}$ & Spectrophotometric & $\begin{array}{l}\text { Abd Alla and Salem } \\
(2020)\end{array}$ \\
\hline Isozeaxanthin & & & & $\begin{array}{l}38.06-265.40 \\
\mathrm{mg} / \mathrm{g}\end{array}$ & & $\begin{array}{l}\text { Abd Alla and Salem } \\
(2020)\end{array}$ \\
\hline Lactucaxanthin & & & & $\begin{array}{l}31.71-97.94 \\
\mathrm{mg} / \mathrm{g}\end{array}$ & & $\begin{array}{l}\text { Abd Alla and Salem } \\
(2020)\end{array}$ \\
\hline Neoxanthin & & & & $\begin{array}{l}44.53-72.19 \\
\mathrm{mg} / \mathrm{g}\end{array}$ & & $\begin{array}{l}\text { Abd Alla and Salem } \\
(2020)\end{array}$ \\
\hline Violaxanthin & & & & $\begin{array}{l}48.32-105.99 \\
\mathrm{mg} / \mathrm{g}\end{array}$ & & $\begin{array}{l}\text { Abd Alla and Salem } \\
(2020)\end{array}$ \\
\hline Antheraxanthin & & & & $\begin{array}{l}40.12-91.46 \\
\mathrm{mg} / \mathrm{g}\end{array}$ & & $\begin{array}{l}\text { Abd Alla and Salem } \\
(2020)\end{array}$ \\
\hline Astaxanthin & & & & $\begin{array}{l}36.88-90.12 \\
\mathrm{mg} / \mathrm{g}\end{array}$ & & $\begin{array}{l}\text { Abd Alla and Salem } \\
(2020)\end{array}$ \\
\hline Canthaxanthin & & & & $\begin{array}{l}45.05-96.12 \\
\mathrm{mg} / \mathrm{g}\end{array}$ & & $\begin{array}{l}\text { Abd Alla and Salem } \\
(2020)\end{array}$ \\
\hline
\end{tabular}

n.i., no information. 


\subsection{Phenolic compounds}

Once the phenolic compounds are released from the pollen grains cytoplasm, they can suffer structural changes in the GIT through different mechanisms, for example, enzyme activities, transformation by bacteria in the colon or interactions with macro and micronutrient compounds in the digestive tract, and consequently may cause them to exhibit different biological activities. The digestibility and absorption of phenolic compounds depend on their physicochemical properties (Carbonell-Capella et al., 2014). For instance, its molecular weights may affect its behave differently: low molecular weight phenolic compounds are water-soluble, but those with high molecular weight may be insoluble in water. Furthermore, the conjugation with other compounds, glycosylation, or exposure to oxidation by enzymes such as polyphenol oxidase, are additional factors affecting the digestible and absorption degree of polyphenolic compounds (Stahl et al., 2002).

Dietary flavonoids from plant-based foods are predominantly present as conjugated glycoside, which generally allow them to be absorbed in the small intestine and pass into the circulatory system (Donovan, Manach, Faulks, \& Kroon, 2006). Catechins, in the flavans subgroup, are one exception with partial or no change in the presence of saliva in the first phase of digestion in the mouth and resistant to hydrolysis in the stomach (Rechner, Spencer, Kuhnle, Hahn, \& Rice-Evans, 2001). When occurs, absorption is associated with the hydrolysis of the glycosylates structure and the release of aglycons as a result of the lactase phlorizin hydrolase (LPH) activity in the brush-border of small intestinal epithelial cells. After which, the process is followed by the passage of passive diffusion into the intestinal epithelial cells due to improved lipophilicity and proximity to the cellular membrane (Crozier et al., 2010; Stahl et al., 2002). Another option of hydrolysis is cytosolic $\beta$-glucosidase (CBG), which is found in epithelial cells. In order for hydrolysis to take place, polar glycosides should be transported to epithelial cells with the participation of an active sodium-dependent glucose transporter through the mediation of CBG (Crozier et al., 2009). Therefore, the presence of aglycons in the intestinal epithelial cells after hydrolysis of flavonoid conjugates may be explained in two possible ways: LPH/passive diffusion and CBG/transport (Stahl et al., 2002). Flavonoids and their metabolites that pass into the large intestine without being absorbed in the small intestine can be absorbed here, but the conjugate parts of flavonoids will be fragmented by the bacterial microflora in the large intestine and then exposed to ring fission, resulting in the production of different phenolic acids (Crozier et al., 2010; Donovan et al., 2006).

Quercetin is one of the most common polyphenols compounds in various plant sources including both $\mathrm{BP}$ and $\mathrm{BB}$ and therefore is one of the most researched flavonoids. A study by Hollman, de Vries, van Leeuwen, Mengelers, and Katan (1995) showed that quercetin is better absorbed than quercetin glycosides, mostly due to the different glycosides moieties linked to them which are depending on the sources of quercetin. In the research conducted by Shi and Williamson (2015), the digestibility and bioavailability of quercetin was compared applying naturally glycosylated quercetin (in meal form) and aglycon quercetin (dietary supplement in tablet form) to six male people. Urine samples were collected for $24 \mathrm{~h}$ and the quercetin amount was calculated by liquid chromatography-mass spectrometry. According to the obtained results, $100 \mathrm{~g}$ of onion gives a comparable amount of quercetin to a 500 mg quercetin aglycone supplement. This means that BP and BB, rich in conjugated glycoside quercetin, can reach a similar level of bioavailability in the diet as quercetin aglycone supplement.

As shown in Table 3, also kaempferol is one of the most common bioactive flavonoids in both bee products. Along with quercetin, kaempferol is usually found in the human diet, showing many positive effects on human health. A recent study reported a decrease in necroinflammatory and collagen accumulation after kaempferol injection into mice (Xu et al., 2019). Thus, kaempferol may be an effective agent in the treatment of common diseases such liver fibrosis. Concerning the absorption and excretion of kaempferol, DuPont, Day, Bennett, Mellon, and Kroon (2004) investigated it on four healthy men and four women. In the study, kaempferol showed a better absorption rate than quercetin, even at low doses, being the 3-glucuronide derivative the most dominant form of kaempferol in plasma.

On the other hand, lower molecular weight phenolic acids like gallic acid are more easily absorbed in the intestine than other phenolic compounds. Otherwise, some phenolic acids, such as hydroxycinnamic acid derivatives, which can be in the form of polymers, present a high resistance for penetration through the intestinal cells with LPH or CBG activity, limiting their absorption in the small intestine (Călinoiu \& Vodnar, 2018). Studies on the digestibility and absorption of phenolic acids revealed that the strong bonds that linked these compounds to lipids, organic acids or sugars are broken by the intestinal microflora, contributing to their digestibility (Crozier et al., 2009; Marín, Miguélez, Villar, \& Lombó, 2015; Rechner et al., 2001). Nevertheless, the esterification of chlorogenic acid and caffeic acid reduces the absorption of these phenolic acids, resulting their absorption from the microbial activities in the colon after hydrolysis with esterases (Manach, Williamson, Morand, Scalbert, \& Rémésy, 2005). A study on the metabolites of chlorogenic acid, quercetin-3-O-rutinoside and black tea phenols was conducted by Olthof, Hollman, Buijsman, Van Amelsvoort, and Katan (2003) on 20 healthy people. They found that half of the chlorogenic acid and $43 \%$ of black tea phenols have been converted to hippuric acid, while quercetin-3-O-rutinoside was metabolized to phenylacetic acids (mostly 3-hydroxyphenylacetic acid). Also, traces of phenolic acids and their metabolites were found in the urine after the intake of chlorogenic acid and quercetin-3-O-rutinoside by volunteers without colon. These results clearly show that a significant portion of dietary polyphenols is hydrolyzed to different metabolites by the intestinal microflora.

\subsection{Carotenoids}

The bioavailability of dietary carotenoids depends on internal and external factors, resulting in different absorption values. Events such as $\mathrm{pH}$ change, enzymatic processes, or degradation of the pollen wall during the $\mathrm{BP}$ transformation into $\mathrm{BB}$ by natural fermentation will change the carotenoid content of $\mathrm{BB}$ and consequently its bioavailability. Furthermore, a low proportion of carotenoids have been reported to be bioaccessible (Carbonell-Capella et al., 2014). The dietary carotenoids release from these natural products follows the process of integration of gastric emulsions into lipid droplets. Then, as a result of the activity of bile salts released into the intestinal lumen, lipid droplets are transferred to mixed micelles. After the carotenoids dissolve in micelles, they are absorbed by the intestinal epithelial cells, packed into chylomicrons and introduced into the lymphatic system (Yonekura \& Nagao, 2007). Each of these processes may restrict the bioavailability and absorption rate of carotenoids. The fat amount and type in the surroundings are other important factors affecting the bioavailability of carotenoids, with a minimum amount of fat required for absorption (Fernández-García et al., 2012). Hence, an oily matrix can significantly increase the absorption of carotenoids and may result in a higher bioavailability score (Carbonell-Capella et al., 2014).

There are no in vitro or in vivo studies on the digestion, bioavailability or absorption of dietary carotenoids in $\mathrm{BP}$ and $\mathrm{BB}$, in the literature. But many studies have examined the lipid content of BP and BB in detail, revealing the amount and types of lipids (Bakour et al., 2019; Campos et al., 2008; Mayda et al., 2020; Tomás et al., 2017). Considering that carotenoids bioavailability depends significantly on lipid content, it is likely that both bee products may show a high level of bioaccessibility. However, it is clear that studies on the bioavailability level of carotenoids present in both bee products are needed.

\subsection{Vitamins}

Like carotenoids, vitamin E from natural sources such as BP and BB is 
solubilized into micelles by bile salts and amphipathic lipids before access the lymphatic system (Rigotti, 2007). Many factors have an impact on the absorption of vitamin E, for example, bile acids and pancreatic fluid (Stahl et al., 2002). Also, lipids play an important role in the vitamin $\mathrm{E}$ absorption, depending it on its different types (Rigotti, 2007). Mărgă;oan et al. (2014) showed that medium-chain fatty acids present in BP such as caproic (6:0), caprylic (8:0), capric (10:0) and lauric (12:0) acids, make the vitamin $\mathrm{E}$ absorption more effective than long-chain fatty acids demonstrated in previous studies (Kuksis, Shaikh, \& Hoffman, 1979).

For Vitamin C, a polar molecule with a relatively high molecular weight, the passage through the cell membrane by passive diffusion is not easy. Therefore, the flow of vitamin $\mathrm{C}$ in or out the cell is controlled by different mechanisms. After BP and BB are ingested, vitamin C enters the circulation through membrane proteins like facilitative glucose transporters and sodium vitamin $\mathrm{C}$ cotransporters in the small intestine (Y. Li \& Schellhorn, 2007; Stahl et al., 2002). On the other hand, due to the resistant pollen wall of the $\mathrm{BP}$, the bioaccessibility can be affected if no digestion is observed while passing through the stomach and ileum (Yesiltas et al., 2014). This is partially valid for BB, because this bee product has an increased degree of digestibility by natural fermentation.

\section{Food applications and safety status of bee pollen and bee bread}

\subsection{Legislation and food applications}

Recently, with the reveal of the nutritional and therapeutic properties of BP and BB, expanding interest in these products has emerged. Following the interest, some regulations have been framed individually in many countries, especially regarding the use of BP. For example, Argentina (Artículo 785 - Res 1550, 12.12.90), Brazil (Legislation: Instrução Normativa n.3, de 19 de Janeiro de 2001), China (Legislation: NY 5137-2002 and GB/T 19330-2008), Poland (Legislation: PN-R78893 "Obnoza pylkowe" -Polish legislation for bee-pollen) and Switzerland (Legislation: Swiss Food Manual: Pollen Bienenprodukte, BAG Swiss Federal Office for Public Health) are among the countries with high producing rates of $\mathrm{BP}$ and where related regulations have been made (Ghosh \& Jung, 2017). At international level, aiming to harmonize internationally recognized criteria, the International Standard Organization, ISO, have recently established a working group within the sub-committee ISO/TC 35/SC 19, focused in pollen. These concerning regulations define the sensory requirements (color, taste and smell), physical and chemical indexes (moisture, ash, protein, $\mathrm{pH}$, etc.), microbial limits but also packing and storage conditions. Generally, there are also no established standards concerning the safety and use of BP and even less regulation for $\mathrm{BB}$.

$\mathrm{BP}$ and $\mathrm{BB}$ are usually marketed in their raw form. However, in the last years, with the increase in the supply of functionalized foods, studies have focused on developing new food products by mixing natural sources of bioactive compounds such as $\mathrm{BP}$ and $\mathrm{BB}$, with the final goal of increasing the nutritional value of the food products and thus the positive effects on health, as well as food preservative activity.

Conte, Del Caro, Balestra, Piga, and Fadda (2018) determined the physicochemical and sensory properties of gluten-free bread enriched with polyfloral BP at 1, 2, 3, 4 and 5\%, thus emphasizing that this bread may be placed in the gluten-free products market against the health problems caused by gluten. Krystyjan, Gumul, Ziobro, and Korus (2015) added BP to biscuits to show their potential as dietary supplements and verify that there was no change in the fat content of the biscuits, while there was a significant increase in the content of sugar, protein, fiber, polyphenols and antioxidant potential of biscuits. In a similar study on bakery products, different rates (16\% and $32 \%)$ of BP were mixed with wheat flour to produce cookies. Besides the nutritional, technological and sensory properties of cookies, antioxidant activity was highly linked to the percentage of added pollen (Solgajová, Nôžková, \& Kadáková,
2014). In addition, Anjos, Fernandes, et al. (2019) and Turhan, Saricaoglu, Mortas, Yazici, and Genccelep (2017) demonstrated that BP can be used as a natural antioxidant in meat products, playing a role as anti-lipid oxidation and antimicrobial agent, thereby enabling to extend the shelf life in meat products. Moreover, Yerlikaya (2014) added BP to fermented milk and reported that BP had a positive effect on probiotic viability with no negative effect on the physicochemical properties of fermented milk.

\subsection{Risk in consumption}

BP and BB are natural products of plant origin mainly sold for humans as food supplements, nevertheless there are some concerns about its use for dietary purposes. At the top of these concerns is anxiety that it causes allergic reactions. Normally BP and BB are well tolerated by humans (Bogdanov, 2011), however, there have been reports of some allergic reactions caused by BP, including anaphylaxis and also people suffering from hay fever (Greenberger \& Flais, 2001; Jagdis \& Sussman, 2012). Excluding those people with hay fever, the allergic reactions to those that consume BP is low, with ratios similar to other foods. In a survey of BP allergy in Polish beekeepers and their families, only 2 out of 493 beekeepers received negative reactions after pollen intake and only 22 cases of BP intolerance were seen from the customers of beekeepers, with $0.6 \%$ of BP allergy occurred in family members of beekeepers (Basista, Filipek, \& Sodzawiczny, 2012). More importantly, to this date, there have been no report of serious health problems or deaths due to the use and consumption of bee products, especially BP and BB.

Other factor of risk in this bee products, are the concerns about bacterial contaminants, fungal toxins, or heavy metals and pesticides, which may have a negative impact on health. Collection, processing, or storage conditions of $\mathrm{BP}$ and $\mathrm{BB}$ in unhygienic conditions can provide a suitable environment for microbial growth. The consumption of fresh BP is not generally recommended, yet dry BP is known to be microbiologically safe (Mauriello, De Prisco, Di Prisco, La Storia, \& Caprio, 2017). The status for $\mathrm{BB}$ is different because it is a natural fermented product with a lower $\mathrm{pH}$ and a higher lactic acid content compared to BP, which enables higher resistance to possible microbial contaminations (Khalifa et al., 2020).

Pesticides and heavy metals are among the main pollutants in BP and $\mathrm{BB}$, coming from the environment and from agricultural practices (de Oliveira, do Nascimento Queiroz, da Luz, Porto, \& Rath, 2016). Researches on BP and BB samples obtained in different geographical regions around the globe described pesticide residues at different levels in both bee products (de Oliveira et al., 2016). Around two hundred samples of BP and BB obtained from different apiaries and in different seasons across China were tested for pesticide residues with different types of pesticides detected at different concentrations, with the higher contamination found in BP (Tong et al., 2018). A similar study in Luxemburg enable also the detection of more than one hundred pesticides residues in BP (Beyer et al., 2018), but similar occurrences were also reported in North and South America, Asia and Europe (de Oliveira et al., 2016; Mullin et al., 2010; Roszko, Kamińska, Szymczyk, \& Jędrzejczak, 2016). More important, pesticides are known to cause negative effects not only in human health (dermatological, neurological, carcinogenic, respiratory, and reproductive systems) (Nicolopoulou-Stamati, Maipas, Kotampasi, Stamatis, \& Hens, 2016), but also in honey bees even at sub-lethal dosage (Pettis, Johnson, \& Dively, 2012). Concerning the presence of heavy metals, the study conducted by Dinkov and Stratev (2016), identified the presence of lead and cadmium in BP, which was corroborated by several other studies in BP and BB (Harmanescu, Bordean, \& Gergen, 2007; Zhelyazkova, 2018), but in general with values below the maximum residue limit. However, the risk of BP and $\mathrm{BB}$ consumption in terms of microbiological and environmental contaminants is present, it is not at a level that can affect human health considering the reported studies. 


\section{Future perspective and conclusion}

Concerns over synthetic food additives and consumer demand for a more balanced and healthy diet may have triggered the acceleration of studies on natural products like BP and BB over the past decade. Therefore, researchers are making an effort to reveal its nutritional value and health-promoting properties. However, the amount of studies addressing the mechanisms of interaction of $\mathrm{BP}$ and $\mathrm{BB}$ constituents with the human body and its link with the medical and pharmacological action is still scarce. In the future, to meet this demand in the and to expand the field of technological application of these two bee products, some points should be taken in account: i) research and classification of nutritional and biological activities based on botanical and geographical origin, ii) BP and BB standardization using techniques with higher accuracy and precision, and internationally recognized, iii) developing innovative approaches to increase the bioavailability, especially for BP, and iv) focused studies on the transformation and absorption/assimilation in the digestive system following the ingestion, and their bioactivity, will greatly contribute to the development of both bee products.

In brief, $\mathrm{BP}$ and $\mathrm{BB}$ present a variety of dietary phytochemical compounds with functional properties such as carotenoids, vitamins, phenolic acids and especially flavonoids. Therefore, considering the food industry and the positive effects on human health, these bee products, which have tremendous potential for the production and use as natural and functional ingredients, offer a wide field of study.

\section{Funding}

The authors are grateful to the Foundation for Science and Technology (FCT, Portugal) for financial support, through national funds FCT/MCTES, to CIMO (UIDB/00690/2020). Thanks to the Programa Apícola Nacional 2020-2022 (National Beekeeping Program) for funding the project "NormBee-Standardization of production procedures and quality parameters of bee products" and to Project PDR2020-1.0.1FEADER-031734: "DivInA-Diversification and Innovation on Beekeeping Production”. National funding by FCT- Foundation for Science and Technology, through the institutional scientific employment program-contract with Soraia I. Falcão.

\section{References}

Abbas, M., Saeed, F., Anjum, F. M., Afzaal, M., Tufail, T., Bashir, M. S., et al. (2017) Natural polyphenols: An overview. International Journal of Food Properties, 20(8), 1689-1699.

Abd Alla, A. E., \& Salem, R. A. (2020). Impact of storage period on different types of bee pollen pigments. Journal of Plant Protection and Pathology, 11(1), 9-13.

AbdElsalam, E., Foda, H. S., Abdel-Aziz, M. S., \& Abd, F. K. (2018). Antioxidant and antimicrobial activities of Egyptian bee pollen. Sciences, 8, 1248-1255, 04.

Abouda, Z., Zerdani, I., Kalalou, I., Faid, M., \& Ahami, M. (2011). The antibacterial activity of Moroccan bee bread and bee-pollen (fresh and dried) against pathogenic bacteria. Research Journal of Microbiology, 6(4), 376-384.

Adaškevičiūte, V., Kaškonienè, V., Kaškonas, P., Barčauskaite, K., \& Maruška, A. (2019). Comparison of physicochemical properties of bee pollen with other bee products. Biomolecules, 9(12), 819.

Anjos, O., Fernandes, R., Cardoso, S. M., Delgado, T., Farinha, N., Paula, V., et al. (2019). Bee pollen as a natural antioxidant source to prevent lipid oxidation in black pudding. LWT-Food Science and Technology, 111, 869-875.

Anjos, O., Paula, V., Delgado, T., \& Esrevinho, L. (2019). Influence of the storage conditions on the quality of bee pollen. Zemdirbyste-Agriculture, 106(1), 87-94.

Araújo, J. S., Chambó, E. D., Costa, M. A. P.d. C., da Silva, C., Peixoto, S. M., Lopes de Carvalho, C. A., et al. (2017). Chemical composition and biological activities of mono-and heterofloral bee pollen of different geographical origins. International Journal of Molecular Sciences, 18(5), 921.

Bacha, A. B., Norah, A.-O., Al-Osaimi, M., Harrath, A. H., Mansour, L., \& El-Ansary, A. (2020). The therapeutic and protective effects of bee pollen against prenatal methylmercury induced neurotoxicity in rat pups. Metabolic Brain Disease, 35(1), 215-224.

Bakour, M., Al-Waili, N. S., El Menyiy, N., Imtara, H., Figuira, A. C., Al-Waili, T., et al. (2017). Antioxidant activity and protective effect of bee bread (honey and pollen) in aluminum-induced anemia, elevation of inflammatory makers and hepato-renal toxicity. Journal of Food Science \& Technology, 54(13), 4205-4212.
Bakour, M., Fernandes, Â., Barros, L., Sokovic, M., \& Ferreira, I. C. (2019). Bee bread as a functional product: Chemical composition and bioactive properties. LWT-Food Science and Technology, 109, 276-282.

Baltrušaityte, V., Venskutonis, P. R., \& Čeksterytè, V. (2007). Radical scavenging activity of different floral origin honey and beebread phenolic extracts. Food Chemistry, 101 (2), 502-514.

Bárbara, M. S., Machado, C. S., Sodré, G. D. S., Dias, L. G., Estevinho, L. M., \& De Carvalho, C. A. L. (2015). Microbiological assessment, nutritional characterization and phenolic compounds of bee pollen from Mellipona mandacaia Smith. Molecules, 20(7), 12525-12544, 1983.

Barene, I., Daberte, I., \& Siksna, S. (2015). Investigation of bee bread and development of its dosage forms. Proteins, 24, 20.30-21.70.

Bartkiene, E., Lele, V., Sakiene, V., Zavistanaviciute, P., Zokaityte, E., Dauksiene, A., et al. (2020). Variations of the antimicrobial, antioxidant, sensory attributes and biogenic amines content in Lithuania-derived bee products. LWT-Food Science and Technology, 118, 108793.

Basista, K., Filipek, B., \& Sodzawiczny, K. (2012). Bee pollen allergy in Polish beekeepers and their families. Advances in Dermatology \& Allergology/Postepy Dermatologii i Alergologii, 29(5), 343-347.

Benavides-Guevara, R. M., Quicazan, M. C., \& Ramírez-Toro, C. (2017). Digestibility and availability of nutrients in bee pollen applying different pretreatments. Ingeniería y Competitividad, 19(1), 119-128.

Beyer, M., Lenouvel, A., Guignard, C., Eickermann, M., Clermont, A., Kraus, F., et al. (2018). Pesticide residue profiles in bee bread and pollen samples and the survival of honeybee colonies - a case study from Luxembourg. Environmental Science and Pollution Research, 25(32), 32163-32177.

Bobis, O., Dezmirean, D. S., Marghitas, L. A., Bonta, V., Margaoan, R., Pasca, C., et al. (2017). Beebread from Apis mellifera and Apis dorsata. Comparative chemical composition and bioactivity. Bulletin of University of agricultural Sciences and veterinary medicine Cluj-Napoca. Animal Science and Biotechnologies, 74(1), 43-50.

Bogdanov, S. (2011). Pollen: Nutrition, functional properties, health: A review. Bee Product Science, 1-34.

Bohoyo-Gil, D., Dominguez-Valhondo, D., Garcia-Parra, J., \& González-Gómez, D. (2012). UHPLC as a suitable methodology for the analysis of carotenoids in food matrix. European Food Research and Technology, 235(6), 1055-1061.

Borycka, K., Grabek-Lejko, D., \& Kasprzyk, I. (2015). Antioxidant and antibacterial properties of commercial bee pollen products. Journal of Apicultural Research, 54(5), 491-502.

Bovi, T.d. S., Caeiro, A., dos Santos, S. A. A., Zaluski, R., Shinohara, A. J., Lima, G. P. P., et al. (2020). Seasonal variation of flavonoid content in bee bread: Potential impact on hypopharyngeal gland development in Apis mellifera honey bees. Journal of Apicultural Research, 59(2), 170-177.

Campos, M., Bogdanov, S., de Almeida-Muradian, L. B., Szczesna, T., Mancebo, Y., Frigerio, C., et al. (2008). Pollen composition and standardisation of analytical methods. Journal of Apicultural Research, 47(2), 154-161.

Campos, M., Frigerio, C., Lopes, J., \& Bogdanov, S. (2010). What is the future of BeePollen. Journal of ApiProduct and ApiMedical Science, 2(4), 131-144.

Carbonell-Capella, J. M., Buniowska, M., Barba, F. J., Esteve, M. J., \& Frígola, A. (2014). Analytical methods for determining bioavailability and bioaccessibility of bioactive compounds from fruits and vegetables: A review. Comprehensive Reviews in Food Science and Food Safety, 13(2), 155-171.

Carpes, S., De Alencar, S., Cabral, I., Oldoni, T., Mourão, G. B., Haminiuk, C., et al. (2013). Polyphenols and palynological origin of bee pollen of Apis mellifera L. from Brazil. Characterization of polyphenols of bee pollen. CyTA - Journal of Food, 11(2), $150-161$.

Čeksteryte, V., Kurtinaitienè, B., Venskutonis, P. R., Pukalskas, A., Kazernavičiūtė, R., \& Balžekas, J. (2016). Evaluation of antioxidant activity and flavonoid composition in differently preserved bee products. Czech Journal of Food Sciences, 34(2), 133-142.

Choi, S.-J., Tai, B. H., Cuong, N. M., Kim, Y.-H., \& Jang, H.-D. (2012). Antioxidative and anti-inflammatory effect of quercetin and its glycosides isolated from mampat (Cratoxylum formosum). Food Science and Biotechnology, 21(2), 587-595.

Clement, A., Olatunde, M., Obigwa, P., \& Orijajogun, J. (2017). Effect of drying temperature on nutritional content of Moringa oleifera leave. World Journal of Food Science and Technology, 1(3), 93-96.

Cocan, O., Marghitas, L. A., \& Dezmirean, D. (2006). Total polyphenols, flavonoids and radical scavenging activity of beepollen and beebread collected from Transylvania area. Animal Science and Biotechnologies, 62, 149-152. Bulletin of University of Agricultural Sciences and Veterinary Medicine Cluj-Napoca.

Conte, P., Del Caro, A., Balestra, F., Piga, A., \& Fadda, C. (2018). Bee pollen as a functional ingredient in gluten-free bread: A physical-chemical, technological and sensory approach. LWT-Food Science and Technology, 90, 1-7.

Crane, E. (1992). The world's beekeeping-past and present. In M. Joe (Ed.), Hive and honey bee (pp. 1-22). Graham-Hamilton, Illinois: Dadant\&Sons.

Crozier, A., Del Rio, D., \& Clifford, M. N. (2010). Bioavailability of dietary flavonoids and phenolic compounds. Molecular Aspects of Medicine, 31(6), 446-467.

Crozier, A., Jaganath, I. B., \& Clifford, M. N. (2009). Dietary phenolics: Chemistry, bioavailability and effects on health. Natural Product Reports, 26(8), 1001-1043.

Călinoiu, L. F., \& Vodnar, D. C. (2018). Whole grains and phenolic acids: A review on bioactivity, functionality, health benefits and bioavailability. Nutrients, 10(11), 1615.

Dajas, F. (2012). Life or death: Neuroprotective and anticancer effects of quercetin. Journal of Ethnopharmacology, 143(2), 383-396.

De-Melo, A. A. M., Estevinho, L. M., Moreira, M. M., Delerue-Matos, C., de Freitas, A. d. S., Barth, O. M., et al. (2018). A multivariate approach based on physicochemical parameters and biological potential for the botanical and geographical discrimination of Brazilian bee pollen. Food Bioscience, 25, 91-110. 
De-Melo, A. A. M., Estevinho, L. M., Moreira, M. M., Delerue-Matos, C., Freitas, A.d. S.d., Barth, O. M., et al. (2018). Phenolic profile by HPLC-MS, biological potential, and nutritional value of a promising food: Monofloral bee pollen. Journal of Food Biochemistry, 42(5), Article e12536.

DeGrandi-Hoffman, G., Eckholm, B. J., \& Huang, M. H. (2013). A comparison of bee bread made by Africanized and European honey bees (Apis mellifera) and its effects on hemolymph protein titers. Apidologie, 44(1), 52-63.

Denisow, B., \& Denisow-Pietrzyk, M. (2016). Biological and therapeutic properties of bee pollen: A review. Journal of the Science of Food and Agriculture, 96(13), 4303-4309.

Detry, R., Simon-Delso, N., Bruneau, E., \& Daniel, H.-M. (2020). Specialisation of yeast genera in different phases of bee bread maturation. Microorganisms, 8(11), 1789.

Di Cagno, R., Filannino, P., Cantatore, V., \& Gobbetti, M. (2019). Novel solid-state fermentation of bee-collected pollen emulating the natural fermentation process of bee bread. Food Microbiology, 82, 218-230.

Dinkov, D., \& Stratev, D. (2016). The content of two toxic heavy metals in Bulgarian bee pollen. International Food Research Journal, 23(3), 1343.

Domenici, V., Gabriele, M., Parri, E., Felicioli, A., Sagona, S., Pozzo, L., et al. (2015). Phytochemical composition and antioxidant activity of Tuscan bee pollen of different botanic origins. Italian Journal of Food Science, 27(2), 248-259.

Donovan, J. L., Manach, C., Faulks, R. M., \& Kroon, P. A. (2006). Absorption and metabolism of dietary plant secondary metabolites. In A. Crozier, M. N. Clifford, \& H. Ashihara (Eds.), Plant secondary metabolites (pp. 303-351). Oxford: Blackwell Publishing.

DuPont, M., Day, A., Bennett, R., Mellon, F., \& Kroon, P. (2004). Absorption of kaempferol from endive, a source of kaempferol-3-glucuronide, in humans. European Journal of Clinical Nutrition, 58(6), 947-954.

El Ghouizi, A., El Menyiy, N., Falcão, S. I., Vilas-Boas, M., \& Lyoussi, B. (2020). Chemical composition, antioxidant activity, and diuretic effect of Moroccan fresh bee pollen in rats. Veterinary World, 13(7), 1251-1261.

Eleazu, C., Suleiman, J. B., Othman, Z. A., Zakaria, Z., Nna, V. U., Hussain, N. H. N., et al. (2020). Bee bread attenuates high fat diet induced renal pathology in obese rats via modulation of oxidative stress, downregulation of NF-kB mediated inflammation and Bax signalling. Archives of Physiology and Biochemistry, 1-17.

Evans, J. R., \& Lawrenson, J. G. (2017). Antioxidant vitamin and mineral supplements for preventing age-related macular degeneration. Cochrane Database of Systematic Reviews, 7(7).

Fanali, C., Dugo, L., \& Rocco, A. (2013). Nano-liquid chromatography in nutraceutical analysis: Determination of polyphenols in bee pollen. Journal of Chromatography A, $1313,270-274$.

Farag, S. A., \& El-Rayes, T. (2016). Research article effect of bee-pollen supplementation on performance, Carcass traits and blood parameters of broiler Chickens. Animal and Veterinary Advances, 11(3), 168-177.

Fatrcová-Šramková, K., Nôžková, J., Máriássyová, M., \& Kačániová, M. (2016). Biologically active antimicrobial and antioxidant substances in the Helianthus annuus L. bee pollen. Journal of Environmental Science and Health, Part B, 51(3), 176-181.

Feás, X., Vázquez-Tato, M. P., Estevinho, L., Seijas, J. A., \& Iglesias, A. (2012). Organic bee pollen: Botanical origin, nutritional value, bioactive compounds, antioxidant activity and microbiological quality. Molecules, 17(7), 8359-8377.

Fernández-García, E., Carvajal-Lérida, I., Jarén-Galán, M., Garrido-Fernández, J., PérezGálvez, A., \& Hornero-Méndez, D. (2012). Carotenoids bioavailability from foods: From plant pigments to efficient biological activities. Food Research International, 46 (2), 438-450.

Formato, G., \& Smulders, F. J. (2011). Risk management in primary apicultural production. Part 1: Bee health and disease prevention and associated best practices. Veterinary Quarterly, 31(1), 29-47.

Gardana, C., Del Bo, C., Quicazán, M. C., Corrrea, A. R., \& Simonetti, P. (2018). Nutrients, phytochemicals and botanical origin of commercial bee pollen from different geographical areas. Journal of Food Composition and Analysis, 73, 29-38.

Gey, K. F. (1998). Vitamins E plus C and interacting conutrients required for optimal health. Biofactors, $7(1,2), 113-174$.

Ghosh, S., \& Jung, C. (2017). Nutritional value of bee-collected pollens of hardy kiwi, Actinidia arguta (Actinidiaceae) and oak, Quercus sp.(Fagaceae). Journal of AsiaPacific Entomology, 20(1), 245-251.

Greenberger, P. A., \& Flais, M. J. (2001). Bee pollen-induced anaphylactic reaction in an unknowingly sensitized subject. Annals of Allergy, Asthma, \& Immunology, 86(2), 239-242.

Grosso, G., Bei, R., Mistretta, A., Marventano, S., Calabrese, G., Masuelli, L., et al. (2013). Effects of vitamin C on health: A review of evidence. Frontiers in Bioscience, 18, 1017-1029.

Hämäläinen, M., Nieminen, R., Vuorela, P., Heinonen, M., \& Moilanen, E. (2007). Antiinflammatory effects of flavonoids: Genistein, kaempferol, quercetin, and daidzein inhibit STAT-1 and NF-êB activations, whereas flavone, isorhamnetin, naringenin, and pelargonidin inhibit only NF-êB activation along with their inhibitory effect on iNOS expression and NO production in activated macrophages. Mediators of Inflammation, 1-10, 2007.

Harif Fadzilah, N., Jaapar, M. F., Jajuli, R., \& Wan Omar, W. A. (2017). Total phenolic content, total flavonoid and antioxidant activity of ethanolic bee pollen extracts from three species of Malaysian stingless bee. Journal of Apicultural Research, 56(2), $130-135$.

Harmanescu, M., Bordean, D., \& Gergen, I. (2007). Heavy metals content of bee's pollen from different locations of Romania. Lucrari Stiintifice: Medicina Veterinara, 40, 253-260.

Haydak, M. H., \& Palmer, L. S. (1942). Royal jelly and bee bread as sources of vitamins B1 B2, B6, C and nicotinic and pantothenic acids. Journal of Economic Entomology, 35, 319-320.
Hollman, P. C., de Vries, J. H., van Leeuwen, S. D., Mengelers, M. J., \& Katan, M. B. (1995). Absorption of dietary quercetin glycosides and quercetin in healthy ileostomy volunteers. American Journal of Clinical Nutrition, 62(6), 1276-1282.

Ibrahim, I., Balasundram, S., Abdullah, N., Alias, M., \& Mardan, M. (2012). Morphological characterization of pollen collected by Apis dorsata from a Tropical Rainforest. International Journal of Botany, 8(3), 96-106.

Ivanišová, E., Kačániová, M., Frančáková, H., Petrová, J., Hutková, J., Brovarskyi, V., et al. (2015). Bee bread-perspective source of bioactive compounds for future. Potravinarstvo Slovak Journal of Food Sciences, 9(1), 592-598.

Jagdis, A., \& Sussman, G. (2012). Anaphylaxis from bee pollen supplement. CMAJ Canadian Medical Association Journal, 184(10), 1167-1169.

Karabagias, I. K., Karabagias, V. K., Gatzias, I., \& Riganakos, K. A. (2018). Bio-functional properties of bee pollen: The case of "bee pollen yoghurt". Coatings, 8(12), 423.

Kaškonienè, V., Adaškevičiūte, V., Kaškonas, P., Mickienè, R., \& Maruška, A. (2020). Antimicrobial and antioxidant activities of natural and fermented bee pollen. Food Bioscience, 34, 100532.

Keller, A., Danner, N., Grimmer, G., Ankenbrand, M., von der, Von Der Ohe, K., et al. (2015). Evaluating multiplexed next-generation sequencing as a method in palynology for mixed pollen samples. Plant Biology, 17(2), 558-566.

Ketkar, S. S., Rathore, A. S., Lohidasan, S., Rao, L., Paradkar, A. R., \& Mahadik, K. R. (2014). Investigation of the nutraceutical potential of monofloral Indian mustard bee pollen. Journal of Integrative Medicine, 12(4), 379-389.

Khalifa, S. A., Elashal, M., Kieliszek, M., Ghazala, N. E., Farag, M. A., Saeed, A., et al. (2020). Recent insights into chemical and pharmacological studies of bee bread. Trends in Food Science \& Technology, 97, 300-316.

Kieliszek, M., Piwowarek, K., Kot, A. M., Błażejak, S., Chlebowska-Śmigiel, A., \& Wolska, I. (2018). Pollen and bee bread as new health-oriented products: A review. Trends in Food Science \& Technology, 71, 170-180.

Kostić, A.Ž., Milinčić, D. D., Gašić, U. M., Nedić, N., Stanojević, S. P., Tešić, Ž. L., et al. (2019). Polyphenolic profile and antioxidant properties of bee-collected pollen from sunflower (Helianthus annuus L.) plant. LWT-Food Science and Technology, 112, 108244.

Kowalski, S., \& Lukasiewicz, M. (2017). Application of randomly methylated cyclodextrin in extraction of antioxidant-like compounds from bee bread. Journal of Food \& Nutrition Research, 56(2), 121-128.

Krystyjan, M., Gumul, D., Ziobro, R., \& Korus, A. (2015). The fortification of biscuits with bee pollen and its effect on physicochemical and antioxidant properties in biscuits. LWT-Food Science and Technology, 63(1), 640-646.

Kuksis, A., Shaikh, N. A., \& Hoffman, A. G. (1979). Lipid absorption and metabolism. Environmental Health Perspectives, 33, 45-55.

Kurek-Górecka, A., Górecki, M., Rzepecka-Stojko, A., Balwierz, R., \& Stojko, J. (2020). Bee products in dermatology and skin care. Molecules, 25(3), 556.

Laha, R. C., De Mandal, S., Ralte, L., Ralte, L., Kumar, N. S., Gurusubramanian, G., et al. (2017). Meta-barcoding in combination with palynological inference is a potent diagnostic marker for honey floral composition. AMB Express, 7(1), 1-8.

LeBlanc, B. W., Davis, O. K., Boue, S., DeLucca, A., \& Deeby, T. (2009). Antioxidant activity of Sonoran Desert bee pollen. Food Chemistry, 115(4), 1299-1305.

Li, F.-S., Phyo, P., Jacobowitz, J., Hong, M., \& Weng, J.-K. (2019). The molecular structure of plant sporopollenin. Nature Plants, 5(1), 41-46.

Li, Y., \& Schellhorn, H. E. (2007). New developments and novel therapeutic perspectives for vitamin C. Journal of Nutrition, 137(10), 2171-2184.

Li, C., Yu, W., Wu, P., \& Chen, X. D. (2020). Current in vitro digestion systems for understanding food digestion in human upper gastrointestinal tract. Trends in Food Science \& Technology, 96, 114-126.

Manach, C., Williamson, G., Morand, C., Scalbert, A., \& Rémésy, C. (2005). Bioavailability and bioefficacy of polyphenols in humans. I. Review of 97 bioavailability studies. American Journal of Clinical Nutrition, 81(1), 230S-242S.

Marín, L., Miguélez, E. M., Villar, C. J., \& Lombó, F. (2015). Bioavailability of dietary polyphenols and gut microbiota metabolism: Antimicrobial properties. BioMed Research International, 1-18, 2015.

Markiewicz-Żukowska, R., Naliwajko, S. K., Bartosiuk, E., Moskwa, J., Isidorov, V., Soroczyńska, J., et al. (2013). Chemical composition and antioxidant activity of beebread, and its influence on the glioblastoma cell line (U87MG). Journal of Apicultural Science, 57(2), 147-157.

Martins, M. C., Morgano, M. A., Vicente, E., Baggio, S. R., \& Rodriguez-Amaya, D. B. (2011). Physicochemical composition of bee pollen from eleven Brazilian states. Journal of Apicultural Science, 55(2), 107-116.

Mauriello, G., De Prisco, A., Di Prisco, G., La Storia, A., \& Caprio, E. (2017). Microbial characterization of bee pollen from the Vesuvius area collected by using three different traps. PloS One, 12(9), Article e0183208.

Mayda, N., Özkök, A., Bayram, N. E., Gerçek, Y. C., \& Sorkun, K. (2020). Bee bread and bee pollen of different plant sources: Determination of phenolic content, antioxidant activity, fatty acid and element profiles. Journal of Food Measurement and Characterization, 14(4), 1795-1809.

Melo, I. L. P.d., \& Almeida-Muradian, L. B.d. (2010). Stability of antioxidants vitamins in bee pollen samples. Química Nova, 33(3), 514-518.

Mizrahi, A., \& Lensky, Y. (2013). Bee products: Properties, applications, and apitherapy. Springer Science \& Business Media.

Moita, E., Sousa, C., Andrade, P. B., Fernandes, F., Pinho, B. R., Silva, L. R., et al. (2014). Effects of Echium plantagineum L. Bee pollen on basophil degranulation: Relationship with metabolic profile. Molecules, 19(7), 10635-10649.

Mullin, C. A., Frazier, M., Frazier, J. L., Ashcraft, S., Simonds, R., \& Pettis, J. S. (2010). High levels of miticides and agrochemicals in North American apiaries: Implications for honey bee health. PloS One, 5(3), Article e9754. 
Mărgăoan, R., Cornea-Cipcigan, M., Topal, E., \& Kösoğlu, M. (2020). Impact of fermentation processes on the bioactive profile and health-promoting properties of bee bread, mead and honey vinegar. Processes, 8(9), 1081.

Mărgăoan, R., Mărghitaş, L. A., Dezmirean, D. S., Dulf, F. V., Bunea, A., Socaci, S. A.a., et al. (2014). Predominant and secondary pollen botanical origins influence the carotenoid and fatty acid profile in fresh honeybee-collected pollen. Journal of Agricultural and Food Chemistry, 62(27), 6306-6316.

Mărgăoan, R., Stranț, M., Varadi, A., Topal, E., Yücel, B., Cornea-Cipcigan, M., et al. (2019). Bee collected pollen and bee bread: Bioactive constituents and health benefits. Antioxidants, 8(12), 568.

Negri, G., Barreto, L. M. R. C., Sper, F. L., Carvalho, C.d., \& Campos, M.d. G. R. (2018) Phytochemical analysis and botanical origin of Apis mellifera bee pollen from the municipality of Canavieiras, Bahia State, Brazil. Brazilian Journal of Food Technology, 21, Article e2016176.

Nicolopoulou-Stamati, P., Maipas, S., Kotampasi, C., Stamatis, P., \& Hens, L. (2016). Chemical pesticides and human health: The urgent need for a new concept in agriculture. Frontiers in Public Health, 4, 148.

de Oliveira, R. C., do Nascimento Queiroz, S. C., da Luz, C. F. P., Porto, R. S., \& Rath, S. (2016). Bee pollen as a bioindicator of environmental pesticide contamination. Chemosphere, 163, 525-534.

Oliveira, K. C., Moriya, M., Azedo, R. A., Almeida-Muradian, L. B.d., Teixeira, E. W., Alves, M. L., et al. (2009). Relationship between botanical origin and antioxidants vitamins of bee-collected pollen. Química Nova, 32(5), 1099-1102.

Olthof, M. R., Hollman, P. C., Buijsman, M. N., Van Amelsvoort, J. M., \& Katan, M. B. (2003). Chlorogenic acid, quercetin-3-rutinoside and black tea phenols are extensively metabolized in humans. Journal of Nutrition, 133(6), 1806-1814.

Othman, Z. A., Ghazali, W., Syaheedah, W., Noordin, L., Yusof, M., Aiman, N., et al. (2020). Phenolic compounds and the anti-atherogenic effect of bee bread in high-fat diet-induced obese rats. Antioxidants, 9(1), 33.

Othman, Z. A., Noordin, L., Ghazali, W. S. W., Omar, N., \& Mohamed, M. (2019). Nutritional, phytochemical and antioxidant analysis of bee bread from different regions of Malaysia. Indian Journal of Pharmaceutical Sciences, 81(5), 955-960.

Pascoal, A., Rodrigues, S., Teixeira, A., Feás, X., \& Estevinho, L. M. (2014). Biological activities of commercial bee pollens: Antimicrobial, antimutagenic, antioxidant and anti-inflammatory. Food and Chemical Toxicology, 63, 233-239.

Pettis, J. S., Johnson, J., \& Dively, G. (2012). Pesticide exposure in honey bees results in increased levels of the gut pathogen Nosema. Naturwissenschaften, 99(2), 153-158.

Rechner, A. R., Spencer, J. P., Kuhnle, G., Hahn, U., \& Rice-Evans, C. A. (2001). Novel biomarkers of the metabolism of caffeic acid derivatives in vivo. Free Radical Biology and Medicine, 30(11), 1213-1222.

Rigotti, A. (2007). Absorption, transport, and tissue delivery of vitamin E. Molecular Aspects of Medicine, 28(5-6), 423-436.

Rizvi, S., Raza, S. T., Faizal Ahmed, A. A., Abbas, S., \& Mahdi, F. (2014). The role of vitamin E in human health and some diseases. Sultan Qaboos University Medical Journal, 14(2), e157.

Rocchetti, G., Castiglioni, S., Maldarizzi, G., Carloni, P., \& Lucini, L. (2019). UHPLC-ESI QTOF-MS phenolic profiling and antioxidant capacity of bee pollen from different botanical origin. International Journal of Food Science and Technology, 54(2), $335-346$.

Ross, A. C. (2010). Vitamin a. In Bioactive compounds and cancer (pp. 335-356). Springer.

Roszko, M.Ł., Kamińska, M., Szymczyk, K., \& Jędrzejczak, R. (2016). Levels of selected persistent organic pollutants (PCB, PBDE) and pesticides in honey bee pollen sampled in Poland. PloS One, 11(12), Article e0167487.

Russell, R., Beard, J. L., Cousins, R. J., Dunn, J. T., Ferland, G., Hambidge, K., et al. (2001). Dietary reference intakes for vitamin A, vitamin K, arsenic, boron, chromium, copper, iodine, iron, manganese, molybdenum, nickel, silicon, vanadium, and zinc. Journal of the American Dietetic Association, 101, 294-301.

Rzepecka-Stojko, A., Stojko, J., Kurek-Górecka, A., Górecki, M., Kabała-Dzik, A., Kubina, R., et al. (2015). Polyphenols from bee pollen: Structure, absorption, metabolism and biological activity. Molecules, 20(12), 21732-21749.

Rzepecka-Stojko, A., Stojko, J., Kurek-Górecka, A., Górecki, M., Sobczak, A., Stojko, R., et al. (2015). Polyphenol content and antioxidant activity of bee pollen extracts from Poland. Journal of Apicultural Research, 54(5), 482-490.

Salazar-González, C., \& Díaz-Moreno, C. (2016). The nutritional and bioactive aptitude of bee pollen for a solid-state fermentation process. Journal of Apicultural Research, 55 (2), 161-175.

Sattler, J. A. G., de Melo, I. L. P., Granato, D., Araújo, E., de Freitas, A.d. S., Barth, O. M., et al. (2015). Impact of origin on bioactive compounds and nutritional composition of bee pollen from southern Brazil: A screening study. Food Research International, 77, 82-91.

Selamoglu, Z. (2017). Honeybee pollen with health beneficial: An update. Journal of Traditional Medicine \& Clinical Naturopathy, 7(1), e141.

Sesso, H. D., Buring, J. E., Christen, W. G., Kurth, T., Belanger, C., MacFadyen, J., et al. (2008). Vitamins $\mathrm{E}$ and $\mathrm{C}$ in the prevention of cardiovascular disease in men: The physicians' health study II randomized controlled trial. Jama, 300(18), 2123-2133.

Shi, Y., \& Williamson, G. (2015). Comparison of the urinary excretion of quercetin glycosides from red onion and aglycone from dietary supplements in healthy subjects: A randomized, single-blinded, cross-over study. Food \& Function, 6(5), $1443-1448$.

Sobral, F., Calhelha, R. C., Barros, L., Dueñas, M., Tomás, A., Santos-Buelga, C., et al. (2017). Flavonoid composition and antitumor activity of bee bread collected in northeast Portugal. Molecules, 22(2), 248.

Solgajová, M., Nôžková, J., \& Kadáková, M. (2014). Quality of durable cookies enriched with rape bee pollen. Journal of Central European Agriculture, 15(1), 24-38.
Stahl, W., Van Den Berg, H., Arthur, J., Bast, A., Dainty, J., Faulks, R. M., et al. (2002). Bioavailability and metabolism. Molecular Aspects of Medicine, 23(1-3), 39.

Stanciu, O., Marghitas, L. A., \& Dezmirean, D. (2007). Examination of antioxidant capacity of beebread extracts by different complementary assays. Bulletin of University of Agricultural Sciences and Veterinary Medicine Cluj-Napoca-Animal Science and Biotechnologies, 64(1-2).

Stanciu, O., Marghitas, L. A., \& Dezmirean, D. (2008). A comparison of methods used to define the antioxidant capacity of bee pollen and beebread from Romania. In Paper presented at the proceedings. 43rd Croatian and 3rd international symposium on agriculture. Opatija. Croatia.

Sun, L., Guo, Y., Zhang, Y., \& Zhuang, Y. (2017). Antioxidant and anti-tyrosinase activities of phenolic extracts from rape bee pollen and inhibitory melanogenesis by cAMP/MITF/TYR pathway in B16 mouse melanoma cells. Frontiers in Pharmacology, $8,104$.

Tavdidishvili, D., Khutsidze, T., Pkhakadze, M., Vanidze, M., \& Kalandia, A. (2014), Flavonoids in Georgian bee bread and bee pollen. Journal of Chemistry and Chemical Engineering, 8(7), 676-681.

Thakur, M., \& Nanda, V. (2020). Composition and functionality of bee pollen: A review. Trends in Food Science \& Technology, 98, 82-106.

Tomás, A., Falcão, S. I., Russo-Almeida, P., \& Vilas-Boas, M. (2017). Potentialities of beebread as a food supplement and source of nutraceuticals: Botanical origin, nutritional composition and antioxidant activity. Journal of Apicultural Research, 56 (3), 219-230.

Tong, Z., Duan, J., Wu, Y., Liu, Q., He, Q., Shi, Y., et al. (2018). A survey of multiple pesticide residues in pollen and beebread collected in China. The Science of the Total Environment, 640, 1578-1586.

Tsao, R. (2010). Chemistry and biochemistry of dietary polyphenols. Nutrients, 2(12), 1231-1246.

Turhan, S., Saricaoglu, F. T., Mortas, M., Yazici, F., \& Genccelep, H. (2017). Evaluation of color, lipid oxidation and microbial quality in meatballs formulated with bee pollen during frozen storage. Journal of Food Processing and Preservation, 41(3), Article e12916.

T'ai, H. R., \& Cane, J. H. (2000). Pollen nutritional content and digestibility for animals. In Pollen and pollination (pp. 187-209). Springer.

Urcan, A. C., Criste, A. D., Dezmirean, D. S., Mărgăoan, R., Caeiro, A., \& Graça Campos, M. (2018). Similarity of data from bee bread with the same taxa collected in India and Romania. Molecules, 23(10), 2491.

Xu, T., Huang, S., Huang, Q., Ming, Z., Wang, M., Li, R., et al. (2019). Kaempferol attenuates liver fibrosis by inhibiting activin receptor-like kinase 5. Journal of Cellular and Molecular Medicine, 23(9), 6403-6410.

Yang, J. H., Shin, B. Y., Han, J. Y., Kim, M. G., Wi, J. E., Kim, Y. W., et al. (2014). Isorhamnetin protects against oxidative stress by activating Nrf2 and inducing the expression of its target genes. Toxicology and Applied Pharmacology, 274(2), 293-301.

Yang, K., Wu, D., Ye, X., Liu, D., Chen, J., \& Sun, P. (2013). Characterization of chemical composition of bee pollen in China. Journal of Agricultural and Food Chemistry, 61(3), 708-718.

Yan, S., Li, Q., Xue, X., Wang, K., Zhao, L., \& Wu, L. (2019). Analysis of improved nutritional composition of bee pollen (Brassica campestris L.) after different fermentation treatments. International Journal of Food Science and Technology, 54(6), 2169-2181.

Yerlikaya, O. (2014). Effect of bee pollen supplement on antimicrobial, chemical, rheological, sensorial properties and probiotic viability of fermented milk beverages. Mljekarstvo, 64(4), 268-279.

Yesiltas, B., Capanoglu, E., Firatligil-Durmus, E., Sunay, A. E., Samanci, T., \& Boyacioglu, D. (2014). Investigating the in-vitro bioaccessibility of propolis and pollen using a simulated gastrointestinal digestion System. Journal of Apicultural Research, 53(1), 101-108.

Yonekura, L., \& Nagao, A. (2007). Intestinal absorption of dietary carotenoids. Molecular Nutrition \& Food Research, 51(1), 107-115.

Zang, Y., Zhang, L., Igarashi, K., \& Yu, C. (2015). The anti-obesity and anti-diabetic effects of kaempferol glycosides from unripe soybean leaves in high-fat-diet mice. Food \& Function, 6(3), 834-841.

Zhang, Z., Cao, H., Chen, C., Chen, X., Wei, Q., \& Zhao, F. (2017). Effects of fermentation by Ganoderma lucidum and Saccharomyces cerevisiae on rape pollen morphology and its wall. Journal of Food Science \& Technology, 54(12), 4026-4034.

Zhang, Y., Zhu, X., Huang, T., Chen, L., Liu, Y., Li, Q., et al. (2016). $\beta$-Carotene synergistically enhances the anti-tumor effect of 5-fluorouracil on esophageal squamous cell carcinoma in vivo and in vitro. Toxicology Letters, 261, 49-58.

Zhelyazkova, I. (2018). Assessment of heavy metals content in pollen from the combs (bee bread) in regions with different anthropogenic impact. Macedonian Journal of Animal Science, 8(1), 47-53.

Zuluaga-Domínguez, C., Serrato-Bermudez, J., \& Quicazán, M. (2018). Influence of drying-related operations on microbiological, structural and physicochemical aspects for processing of bee-pollen. Engineering in Agriculture, Environment and Food, 11(2), 57-64.

Zuluaga, C. M., Serrato, J. C., \& Quicazana, M. C. (2015a). Chemical, nutritional and bioactive characterization of Colombian bee-bread. Chemical Engineering, 43, 175-180.

Zuluaga, C. M., Serrato, J. C., \& Quicazan, M. C. (2015b). Bee-pollen structure modification by physical and biotechnological processing: Influence on the availability of nutrients and bioactive compounds. Chemical Engineering Transactions, $43,79-84$. 